\title{
Transcriptional mechanism of IRF8 and PU.1 governs microglial activation in neurodegenerative condition
}

\author{
Nan Zhou ${ }^{6}$, Kaili Liu ${ }^{3}$, Yue Sun ${ }^{1,4}$, Ying Cao ${ }^{1,3,5}$, Jing Yang ${ }^{1,2,3,4 \bowtie}$ \\ 1 State Key Laboratory of Membrane Biology, Peking University, Beijing 100871, China \\ 2 IDG/McGovern Institute for Brain Research, Peking University, Beijing 100871, China \\ ${ }^{3}$ Center for Life Sciences, Peking University, Beijing 100871, China \\ ${ }^{4}$ School of Life Sciences, Peking University, Beijing 100871, China \\ ${ }^{5}$ Academy for Advanced Interdisciplinary Studies, Peking University, Beijing 100871, China \\ ${ }^{6}$ School of Medicine, Tsinghua University, Beijing 100084, China \\ $\triangle$ Correspondence: jing.yang@pku.edu.cn (J. Yang)
}

Received September 4, 2018 Accepted October 31, 2018

\begin{abstract}
Microglial activation occurs in divergent neuropathological conditions. Such microglial event has the key involvement in the progression of CNS diseases. However, the transcriptional mechanism governing microglial activation remains poorly understood. Here, we investigate the microglial response to traumatic injuryinduced neurodegeneration by the 3D fluorescence imaging technique. We show that transcription factors IRF8 and PU.1 are both indispensible for microglial activation, as their specific post-developmental deletion in microglia abolishes the process. Mechanistically, we reveal that IRF8 and PU.1 directly target the gene transcription of each other in a positive feedback to sustain their highly enhanced expression during microglial activation. Moreover, IRF8 and PU.1 dictate the microglial response by cooperatively acting through the composite IRF-ETS motifs that are specifically enriched on microglial activation-related genes. This action of cooperative transcription can be further verified biochemically by the synergetic binding of IRF8 and PU.1 proteins to the composite-motif DNA. Our study has therefore elucidated the central transcriptional
\end{abstract}

Nan Zhou, Kaili Liu, Yue Sun and Ying Cao have contributed equally to this work.

Electronic supplementary material The online version of this article (https://doi.org/10.1007/s13238-018-0599-3) contains supplementary material, which is available to authorized users. mechanism of microglial activation in response to neurodegenerative condition.

KEYWORDS microglia, 3D fluorescence imaging technique, neurodegeneration, IRF8, PU.1

\section{INTRODUCTION}

As the specialized immune cells of the central nervous system (CNS), microglia participate in diverse physiological and disease processes. Unlike other glial types, microglia originate from the myeloid-lineage progenitor cells that migrate from the yolk sac into the CNS during the early embryonic stage (Ginhoux et al., 2013; Nayak et al., 2014; Ginhoux and Prinz, 2015; Tay et al., 2016). Microglia exert essential functions in brain development, e.g., in the precise establishment of neural connections (Stephan et al., 2012; Aguzzi et al., 2013; Kettenmann et al., 2013; Schafer and Stevens, 2015; Hong et al., 2016). Also, this glial population is indispensible for maintenance of the neural homeostasis throughout adulthood (Prinz and Priller, 2014; MichellRobinson et al., 2015; Prinz et al., 2017; Tay et al., 2017). For instance, microglia are involved in the neural control of energy balance, and their malfunction results in obesity and other metabolic disorders (Thaler et al., 2010; Kalin et al., 2015; Valdearcos et al., 2015).

Besides their physiological functions, microglia are activated in response to divergent neuropathological insults (Rivest, 2009; Ransohoff and Cardona, 2010; Finsen and Owens, 2011; Ransohoff and Brown, 2012). Microglial 
activation has been widely observed in neural injuries, pathogenic infections and neurodegenerative diseases. Activated microglia have long been characterized for their phagocytic clearance of the tissue debris left from neural injuries, which is critical for restricting inflammation and promoting tissue repair (Neumann et al., 2009; Kettenmann et al., 2011; Fu et al., 2014). In addition, microglia are activated to elicit the immune defense for timely containing and eliminating infectious pathogens, e.g., Zika virus and West Nile virus, the failure of which could lead to dreadful consequences (Town et al., 2006; Terry et al., 2012; Lum et al., 2017; Meertens et al., 2017). Furthermore, it has been increasingly appreciated that microglia have the key involvement in neurodegenerative diseases (Perry et al., 2010; Perry and Holmes, 2014; Zuchero and Barres, 2015; Ransohoff, 2016; Colonna and Butovsky, 2017; Wolf et al., 2017). For example, microglia are responsible for the clearance of amyloid peptides, and the deficit of such microglial function could be causative for Alzheimer's disease (Colonna and Wang, 2016; Ulrich et al., 2017). On the other hand, microglia activated by neurodegenerative cues can release a variety of neurotoxic factors, which collaterally damage neural circuits and thus exaggerate the diseases such as Alzheimer's disease, Parkinson's disease, amyotrophic lateral sclerosis and glaucoma (Soto and Howell, 2014; Heppner et al., 2015; Meyer-Luehmann and Prinz, 2015; Ransohoff, 2016; Lall and Baloh, 2017). Our in-depth knowledge of the microglial activation would therefore help reveal therapeutic targets for treatment of the debilitating CNS diseases (Biber et al., 2016; Colonna and Butovsky, 2017; Herz et al., 2017).

Despite extensive studies, one of the central aspects of microglia biology, i.e., the regulation of microglial activation, has been largely uncharacterized. In particular, the transcriptional mechanism governing the transition of resting microglia to their activated state remains to be better understood. In this study, we investigated the microglial response to the traumatic injury-induced neurodegeneration. We exploited the iDISCO (immunolabeling-enabled three-dimensional imaging of solvent-cleared organs) for the 3D fluorescence imaging of microglial activation on the whole-tissue level. We showed that transcription factors IRF8 and PU.1 are both essential for microglial activation, as their specific post-developmental deletion abolishes this process. To explore the underlying transcriptional mechanism, we profiled the genomic landscapes of IRF8 and PU.1 in activated microglia. We revealed that IRF8 and PU.1 directly target the gene transcription of each other, which establishes positive feedback to sustain their highly enhanced expression during microglial activation. Moreover, IRF8 and PU.1 dictate the microglial response by cooperatively acting through the composite IRF-ETS motifs that are specifically enriched on microglial activation-related genes. We further verify such cooperative transcription by demonstrating the synergetic assembly of IRF8 and PU.1 proteins to the composite-motif DNA in the biochemical assays. Altogether, our study has elucidated the central transcriptional mechanism of microglial activation in response to neurodegeneration.

\section{RESULTS}

\section{D fluorescence imaging of microglial activation}

We exploited the model of traumatic injury-induced neurodegeneration to investigate the microglial activation. Axon degeneration after the traumatic nerve injury effectively led to microglial activation in the optic nerves (Yang et al., 2015). To better visualize and accurately quantify this process, we utilized the 3D fluorescence imaging based on the iDISCO technique (Renier et al., 2014). To prove the strength of this imaging approach (Fig. 1A), we firstly visualized the different components of optic nerves. The 3D network of blood vessels, as immunolabeled by the endothelial-cell specific marker PECAM1, was imaged on the whole-tissue level of optic nerves (Fig. 1B). The lymphatic vessels immunolabeled by the lymphatic endothelial marker LYVE1 were also revealed along the pial surface (Fig. $1 \mathrm{C}$ and Movie S1). In addition, three major glial types in the optic nerves could be clearly visualized, i.e., microglia by the specific marker CD11b or lba1 (Fig. 1D and 1G), astrocytes by GFP in Aldh1/1-GFP transgenic mice (Fig. 1E), and oligodendrocytes by the specific marker Olig2 (Fig. 1H).

We next examined the microglial response to neurodegeneration. The retinal ganglion cells were sparsely labeled via the intravitreal injection of tdTomato-expressing AAV2. While the tdTomato-labeled optic axons projected through the control (i.e., uninjured) optic nerves, a striking pattern of neurodegeneration occurred at 5 days post-injury (Figs. 1F, S1A and S1B, and Movie S2). Such neurodegenerative condition strongly induced microglial activation as revealed by the whole-tissue immunolabeling of Iba1, i.e., a network of ramified resting microglia was seen in the control optic nerves but the increased density of microglia together with their ameboid appearance became evident at 5 days postinjury (Figs. 1G, S1A and S1C). Of importance, while Iba1 has been the common marker for microglial activation, we found out that the gene expression of Iba1 (also known as Aif1) was directly targeted by IRF8 and PU.1 (See below). Therefore, we chose the alternative marker CD11b to reliably mark out the microglial population through this study. As an aside, the increased density of oligodendrocytes was also observed under the neurodegenerative condition (Figs. $1 \mathrm{H}$ and S1D). These results have demonstrated the strength of 3D fluorescence imaging in the whole-tissue assessment of microglial activation.

IRF8 and PU.1 act in microglial activation under neurodegeneration condition

It was essential to determine whether microglial activation was induced by the neurodegeneration of optic axons or simply by the traumatic injury per se. The traumatic injury- 

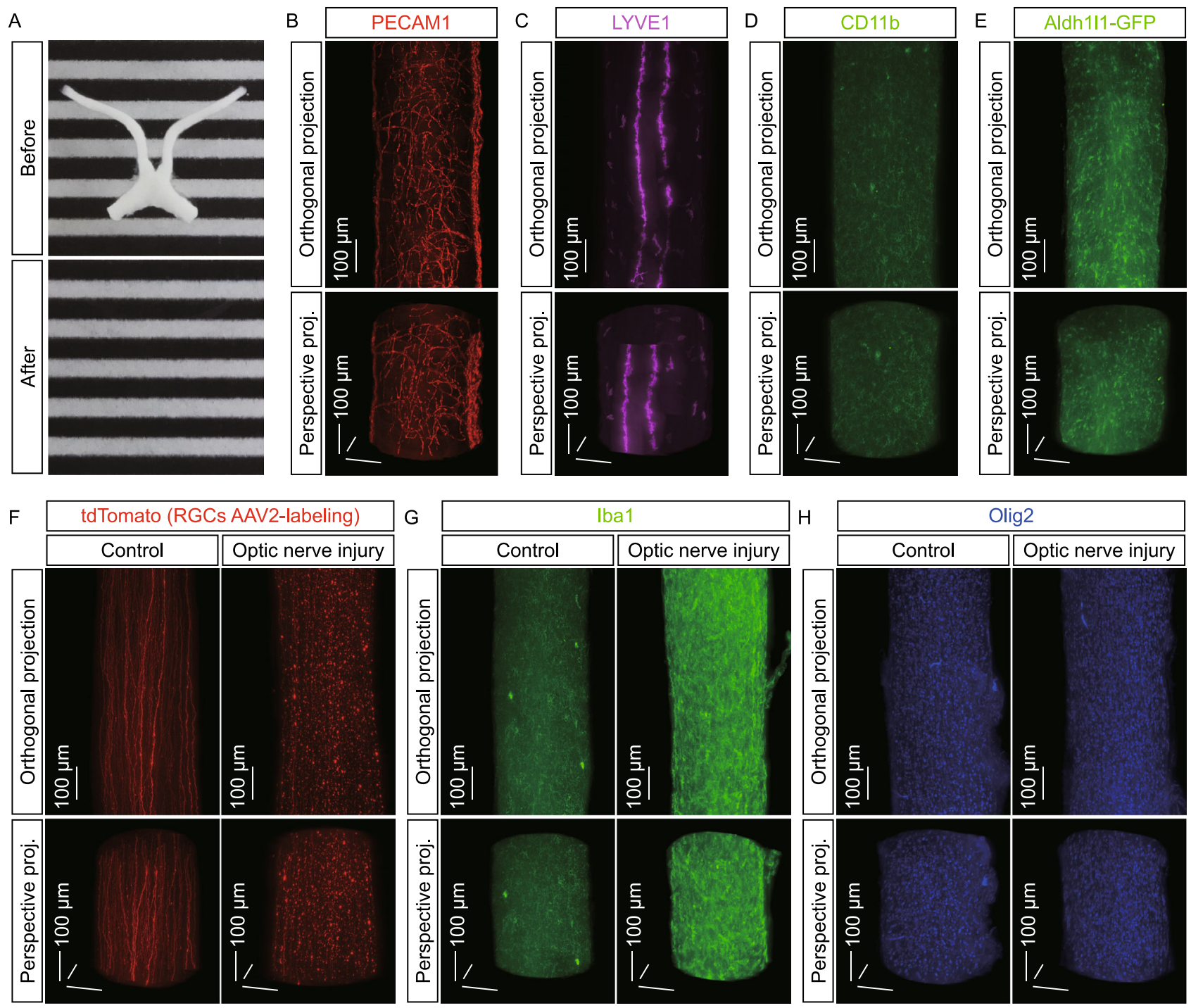

Figure 1. Three-dimensional fluorescence imaging of microglial activation in response to neurodegeneration. (A-E) 3D fluorescence imaging of the mouse optic nerves. (A) The optic nerves before (upper panel) and after (lower panel) the iDISCO procedure. (B-D) The optic nerves of wildtype mice were processed for the whole-tissue immunolabeling of PECAM1 (B), LYVE1 (C) or CD11b (D) and imaged on the lightsheet microscope. (E) The optic nerves of Aldh1/1-GFP transgenic mice were processed for the whole-tissue immunolabeling of GFP and imaged on the lightsheet microscope. Representative orthogonal (upper panels) or perspective (lower panels) 3D-projection images of the optic nerves are shown. (F) 3D fluorescence imaging of the traumatic injuryinduced neurodegeneration. The wildtype mice were intravitreally injected with the tdTomato-expressing AAV2 and then subjected to optic nerve injury. The control (i.e., uninjured) and injured nerves were processed for the whole-tissue immunolabeling of tdTomato and imaged on the lightsheet microscope. Representative orthogonal (upper panels) or perspective (lower panels) 3D-projection images of the optic nerves are shown. $(G$ and $H)$ 3D fluorescence imaging of the glial responses to neurodegeneration. The wildtype mice were subjected to optic nerve injury. The control and injured nerves were processed for the whole-tissue immunolabeling of Iba1 (G) or Olig2 $(\mathrm{H})$ and imaged on the lightsheet microscope. Representative orthogonal (upper panels) or perspective (lower panels) 3D-projection images of the optic nerves are shown.

induced neurodegeneration, including that in the optic nerves, could be suppressed by genetic deletion of Sarm1 (Osterloh et al., 2012; Yang et al., 2015). We observed that microglial activation was largely abolished in Sarm $1^{-/-}$mice compared to that in the control mice (Fig. $2 \mathrm{~A}$ and $2 \mathrm{~B}$ ), suggesting that this microglial response depends on the neurodegenerative condition. We next examined the cellular source involved in microglial activation. Genetic deletion of Cx3cr1 or Ccr2, the two key chemokine receptors for the myeloid-lineage cells, exhibited no detectable effect on 

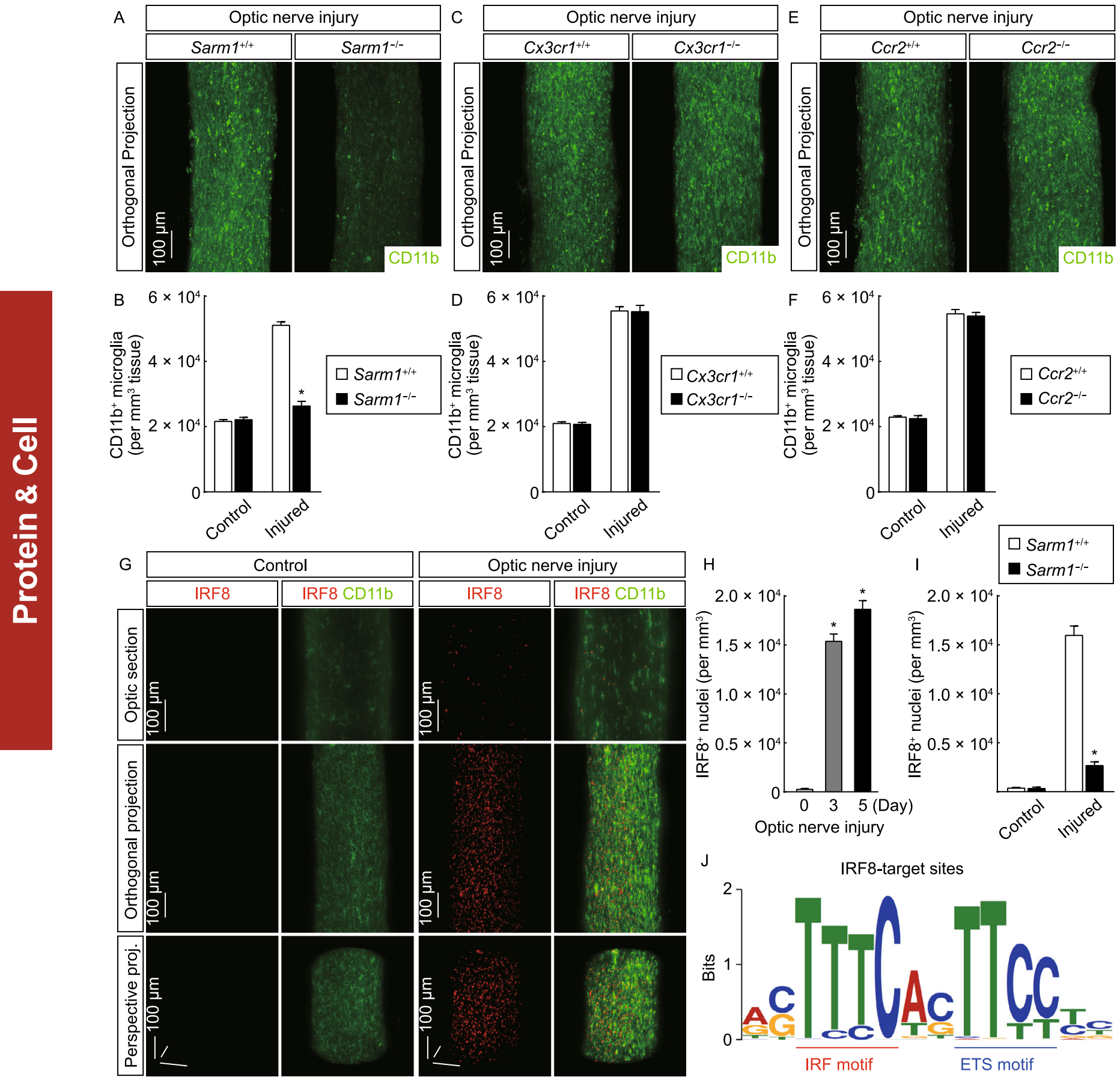

Optic nerve injury
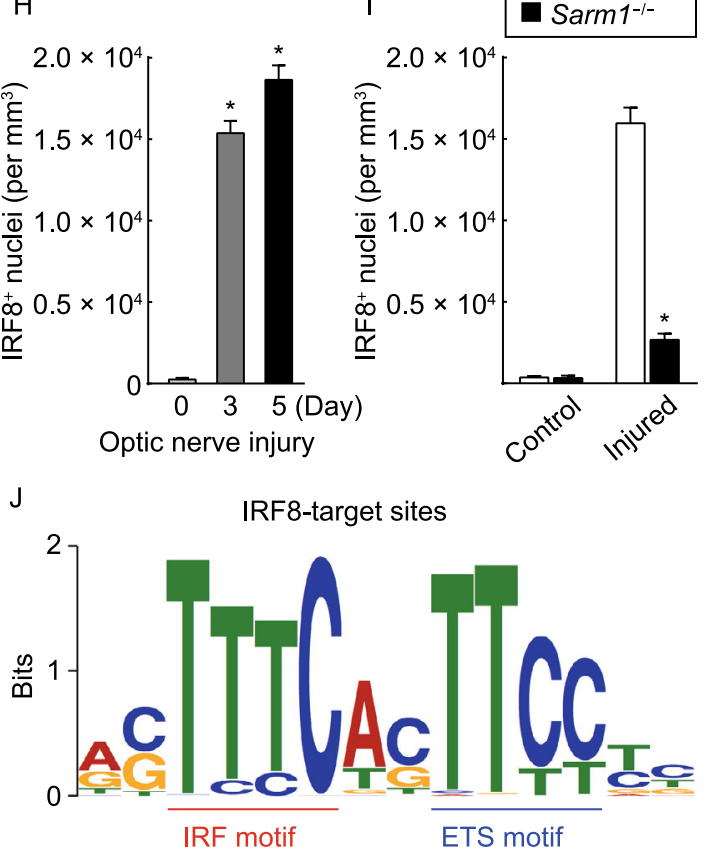
Figure 2. Transcription factor IRF8 acts in microglial activation. (A and B) Microglial activation depends on Sarm1mediated neurodegeneration. Sarm $1^{+/+}$vs. Sarm $1^{-/-}$mice were subjected to optic nerve injury. The control and injured nerves were processed for the whole-tissue immunolabeling of CD11b and imaged on the lightsheet microscope. (A) Representative orthogonal 3D-projection images of the injured nerves. (B) Den-

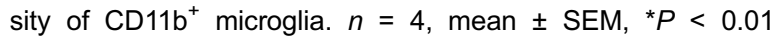
(ANOVA test). (C-F) Microglial activation in the injured optic nerves does not depend on the chemokine receptors $\mathrm{C} \times 3 \mathrm{cr} 1$ and $\mathrm{Ccr} 2 . \mathrm{C} \times 3 \mathrm{Cr}^{+/+}$vs. $\mathrm{C} \times 3 \mathrm{Cr}^{-1-}\left(\mathrm{C}\right.$ and D) or $\mathrm{Ccr}^{+/+}$vs. $\mathrm{Ccr}^{-/-}$ ( $E$ and $F$ ) mice were subjected to optic nerve injury. The control and injured nerves were processed for the whole-tissue immunolabeling of CD11b and imaged on the lightsheet microscope. (C and E) Representative orthogonal 3D-projection images of the injured nerves. ( $D$ and $F$ ) Density of $C D 11 b^{+}$microglia. $n=4$, mean \pm SEM. ( $G$ and $H$ ) Enhanced expression of transcription factor IRF8 in microglial activation. The wildtype mice were subjected to optic nerve injury. The control and injured nerves were processed for the whole-tissue co-immunolabeling of IRF8 (red)/CD11b (green) and imaged on the lightsheet microscope. (G) Representative optical sections of the lightsheet imaging (upper panels), orthogonal 3D projections (middle panels) or perspective $3 D$ projections (lower panels) of the optic nerves. (H) Density of IRF8 ${ }^{+}$nuclei. $n=4$, mean \pm SEM, ${ }^{*} P<0.01$ (Student's $t$-test). (I) IRF8 up-regulation in microglial activation depends on Sarm1-mediated neurodegeneration. Sarm $1^{+/+}$vs. Sarm $1^{-1-}$ mice were subjected to optic nerve injury. The control and injured nerves were processed for the whole-tissue immunolabeling of IRF8 and imaged on the lightsheet microscope. The density of IRF8 ${ }^{+}$nuclei was quantified. $n=3$, mean \pm SEM, ${ }^{*} P<$ 0.01 (ANOVA test). (J) The IRF-ETS composite motif of IRF8 target sites in microglial activation. The wildtype mice were subjected to optic nerve injury, and the injured nerves were processed for ChIP-Seq. The de novo analysis of the composite motif of IRF8 target sites was performed. Positions of the IRF motif and ETS motif are highlighted.

microglial activation in $\mathrm{C} \times 3 \mathrm{Cr}^{-/-}$vs. $\mathrm{C} \times 3 \mathrm{Cr}^{+/+}$mice (Fig. $2 \mathrm{C}$ and $2 \mathrm{D}$ ) or $\mathrm{Ccr}^{-/-}$vs. $\mathrm{Ccr}^{+/+}$mice (Fig. $2 \mathrm{E}$ and $2 \mathrm{~F}$ ), indicating that this process likely does not depend on the recruitment of peripheral macrophages or other precursor cells. To further prove this, we exploited the parabiosis procedure. Previous studies documented that the microglial population in the CNS is $\mathrm{Cx}_{\mathrm{Cr}} 1^{+}$(Goldmann et al., 2013; Parkhurst et al., 2013), which we confirmed in the optic nerves by the complete overlapping of the microglial marker CD11b with the fluorescence EYFP in Cx3cr1 ${ }^{\text {CreERT2-EYFP/+ }}$ mice (Fig. S4A). We performed the parabiosis procedure between the wildtype and $C \times 3 c r 1^{\text {CreERT2-EYFP/+ }}$ mice. The appearance of EYFP-positive cells in the spleen of wildtype mouse in the parabiotic pair showed the success of the procedure (Fig. S1E). However, there were no detectable EYFP-positive cells in the injured optic nerve of the wildtype mouse in the parabiotic pair (Fig. S1F), substantiating that this microglial response does not recruit the circulating population of $\mathrm{C} \times 3 \mathrm{cr} 1^{+}$cells. We then determined whether the increased microglial density would be due to local cell proliferation. There was the low expression of Ki67, the specific marker for proliferating cells, in the control optic nerves, but the significant up-regulation of Ki67 occurred in the injured optic nerves (Fig. S2A and S2B). Moreover, the majority of Ki67-immunolabeled nuclei overlapped with the microglial marker (Fig. S2A), demonstrating the local proliferation of microglia in response to neurodegeneration.

We sought to understand the transcriptional mechanism underlying microglial activation. Prior studies reported that transcription factor IRF8 is involved in microglial activation (Horiuchi et al., 2012; Masuda et al., 2012; Minten et al., 2012). We observed that IRF8 expression was almost undetectable in the control optic nerves by the 3D fluorescence imaging (Fig. $2 \mathrm{G}$ and $2 \mathrm{H}$ ). In contrast, the highly enhanced expression of IRF8 appeared as early as at 3 days post-injury (Fig. 2G and 2H). Notably, the IRF8-immunolabeled nuclei exclusively overlapped with the microglial marker (Fig. 2G). In addition, IRF8 up-regulation was strongly inhibited in the injured optic nerves of Sarm $1^{-1-}$ mice (Fig. 2I), showing that this IRF8 response depends on the neurodegenerative condition. We then set out to profile the genomic landscape of IRF8 in the activated microglia. We optimized the procedure of chromatin immunoprecipitation (ChIP) for nerve tissues without the necessity of cell isolation, taking advantage of the microglia-specific IRF8 expression. The ChIP-Seq analysis successfully identified 851 IRF8 target sites across the genome (Fig. S2C and S2D). Interestingly, through the de novo motif analysis of these IRF8 target sites, we observed not only the consensus interferon regulatory factor (IRF)-binding motif TTTC but also a different, highly represented motif TTCC at two base pairs downstream of the IRF motif (Fig. 2J). In fact, this composite motif (TTCCNNTTTC) was present in $80 \%$ of IRF8 target sites (678 out of 851), implying the cooperative action of IRF8 with an additional transcription factor.

Bioinformatics suggested this composite motif as the potential binding site of the ETS (E26 transformation-specific) family of transcription factors (Sharrocks, 2001; Hollenhorst et al., 2011). We therefore determined the expression profile of these ETS members in the optic nerves. The mRNAs of the majority of ETS members were detectable, and importantly, Pu.1 (also known as Spi1) exhibited the most significant up-regulation after the nerve injury (Fig. 3A). In accordance with this observation, PU.1 expression was low in the control optic nerves but became highly enhanced as early as at 3 days post-injury, as visualized by the $3 \mathrm{D}$ fluorescence imaging (Fig. $3 \mathrm{~B}$ and $3 \mathrm{C}$, and Movie S3). Similar to the microglia-specific expression of IRF8, the PU.1-immunolabeled nuclei also completely overlapped with the microglial marker (Fig. 3B and Movie S3). In addition, PU.1 up-regulation depended on the neurodegenerative 

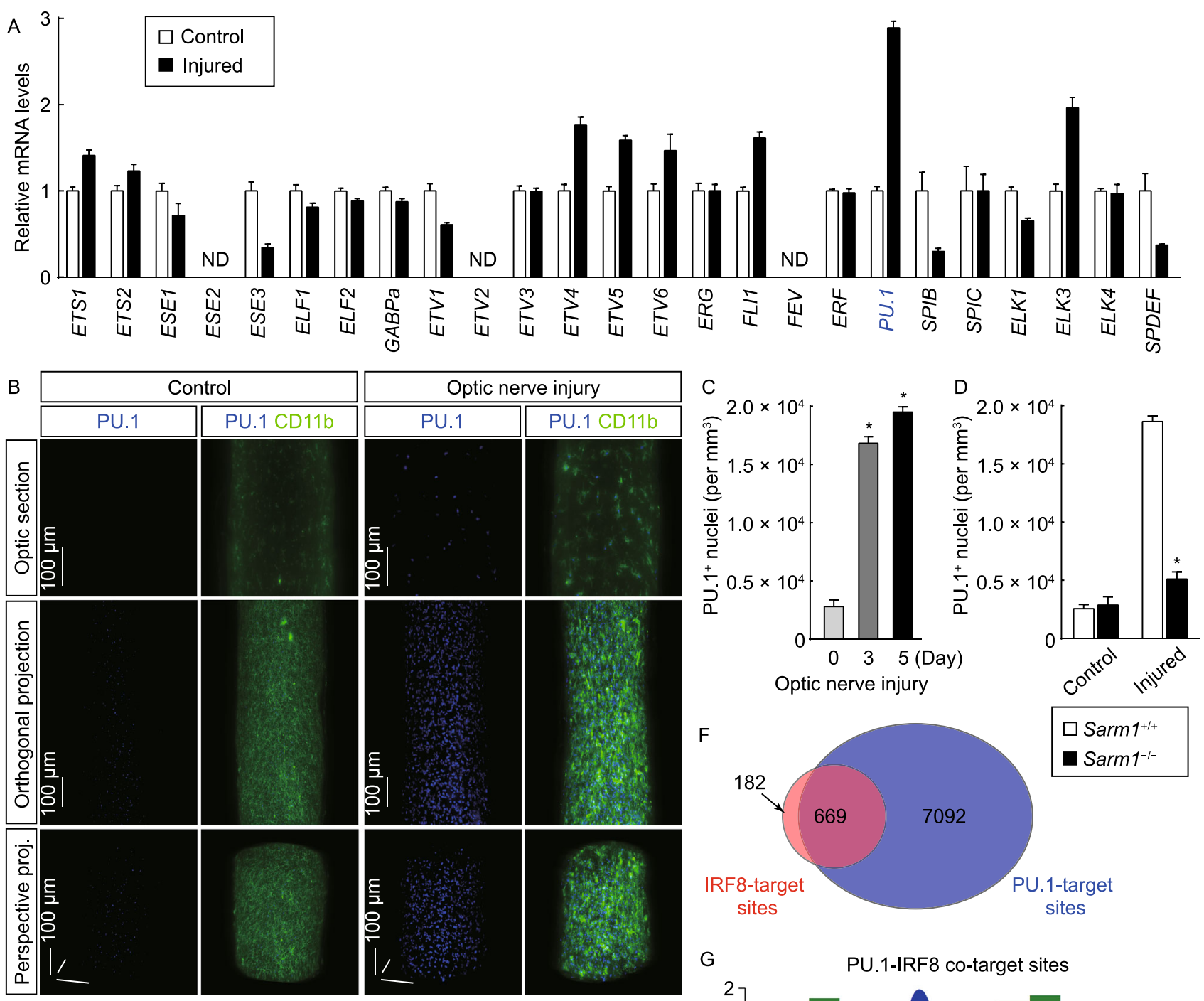

E

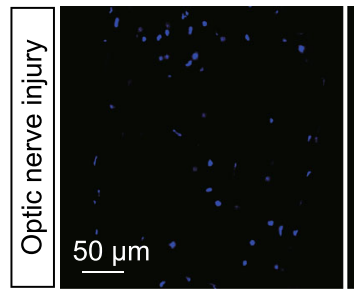

PU.1 IRF8
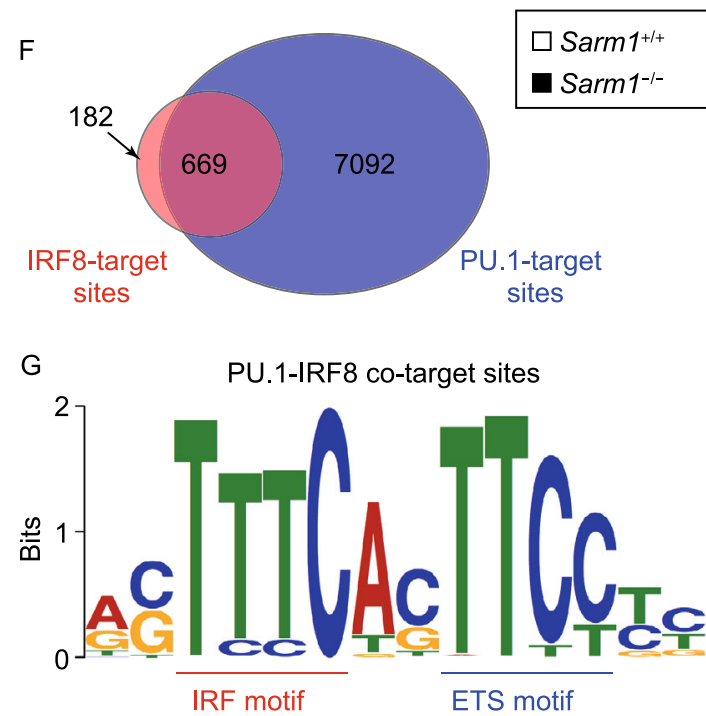
Figure 3. Transcription factor PU.1 participates in microglial activation. (A) Expression of ETS-family transcription factors in the optic nerves. The wildtype mice were subjected to optic nerve injury. The control and injured nerves were processed for the qPCR analysis of ETS-family transcription factors. ND, not detected; $n=4$, mean \pm SEM. (B and C) Enhanced expression of PU.1 in microglial activation. The wildtype mice were subjected to optic nerve injury. The control and injured nerves were processed for the whole-tissue coimmunolabeling of PU.1 (blue)/CD11b (green) and imaged on the lightsheet microscope. (B) Representative optical sections of the lightsheet imaging (upper panels), orthogonal 3D projections (middle panels) or perspective $3 \mathrm{D}$ projections (lower panels) of the optic nerves. (C) Density of PU. $1^{+}$nuclei. $n=4$, mean $\pm \mathrm{SEM}$, ${ }^{*} P<0.01$ (Student's $t$-test). (D) PU.1 upregulation in microglial activation depends on Sarm1-mediated neurodegeneration. Sarm $1^{+/+}$vs. Sarm $1^{-1-}$ mice were subjected to optic nerve injury. The control and injured nerves were processed for the whole-tissue immunolabeling of PU.1 and imaged on the lightsheet microscope. The density of PU. $1^{+}$ nuclei was quantified. $n=3$, mean \pm SEM, ${ }^{*} P<0.01$ (ANOVA test). (E) Co-expression of IRF8 and PU.1 in microglial activation. The wildtype mice were subjected to optic nerve injury, and the injured nerves were examined by immunohistochemistry for IRF8 and PU.1. (F and G) Cooperative transcription of IRF8 and PU.1 in microglial activation. The wildtype mice were subjected to optic nerve injury, and the injured nerves were processed for the ChIP-Seq analysis of PU.1 target sites.

(F) Overlap of PU.1 target sites with the IRF8 target sites.

(G) The composite motif of PU.1 target sites overlapping with IRF8 target sites was analyzed. Positions of the IRF motif and ETS motif are highlighted.

condition, as it was largely abolished in Sarm $1^{-/-}$mice (Fig. 3D).

In light of these results, we hypothesized that PU.1 might be the putative transcription factor cooperating with IRF8 in microglial activation. To support this possibility, we observed that $>90 \%$ of the PU.1-immunolabeled nuclei were positive for IRF8 expression, and vice versa, in the injured optic nerves (Fig. 3E). We then determined the genomic landscape of PU.1 in the activated microglia by the ChIP-Seq procedure, again taking advantage of the microglia-specific PU.1 expression. The ChIP-Seq analysis identified 7,761 PU. 1 target sites across the genome (Fig. S3A and S3B). Strikingly, there was a significant overlap of IRF8 target sites (669 out of 851) with PU.1 target sites (Figs. 3F and S3C). Furthermore, the de novo motif analysis of these PU.1 target sites overlapping with IRF8 target sites resulted in the composite IRF-ETS motif highly resembling that identified from the IRF8 target sites alone (Fig. 3G), suggesting the cooperative transcriptional action of IRF8 and PU. 1 in the microglial response to neurodegeneration.

\section{Cross-regulation of IRF8 and PU.1 in microglial} activation

To decipher the transcriptional mechanism of IRF8 and PU.1, we noticed that there were two PU.1 target sites at the gene locus of Irf8, while there was no detectable IRF8 target site at this locus (Fig. 4A). This observation suggested that PU.1 could directly target IRF8 expression in microglial activation. To test this possibility, we bred CX3cr1 ${ }^{\text {CreERT2-EYFPI+ }}$ P Pu. $1^{\mathrm{fl} / \mathrm{fl}}$ mice to achieve the microgliaspecific deletion of PU.1 in adult mice. The efficiency of the Cre-recombinase activity induced by 4-hydroxytamoxifen was confirmed by the complete overlap of the microglial marker with the Cre-dependent fluorescence tdTomato in Cx3cr1 $1^{\text {CreERT2-EYFP/+} ; ~ R o s a 26-L S L-t d T o m a t o ~}$ mice (Fig. S4B). Of note, we observed the significant decrease of microglial population in the optic nerves, as well as in other CNS regions, about two weeks after the Cre-induction in $\mathrm{Cx} 3 \mathrm{Cr} 1^{\mathrm{CreERT} 2 /+}$; Pu. $1^{\mathrm{fllfl}}$ mice (data not shown). This phenomenon could reflect the microglial death due to the gradual depletion of PU.1 protein after the gene knockout, given the essential function of PU.1 in the myeloid-lineage cells (Scott et al., 1994; McKercher et al., 1996; Kierdorf et al., 2013). To circumvent this issue, the mice were subjected to the optic nerve injury immediately after the daily treatment of 4-hydroxytamoxifen for 6 days. There was robust PU.1 upregulation in the injured optic nerves of control $\mathrm{Cx} 3 \mathrm{Cr}^{+/+}$; Pu. $1^{f l f l}$ mice, but this PU.1 response was abolished in

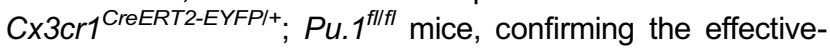
ness of genetic deletion (Fig. 4B and Fig. 4C). More importantly, IRF8 up-regulation was significantly blunted in Cx3Cr1 ${ }^{\text {CreERT2-EYFP/+ }} ; P u .1^{f l f l}$ mice compared to that in control $\mathrm{C} \times 3 \mathrm{Cr}^{+/+} ; \mathrm{Pu} .1^{\mathrm{flfl} f \mathrm{l}}$ mice (Fig. $4 \mathrm{~B}$ and $4 \mathrm{C}$ ), demonstrating that PU.1 regulates IRF8 expression in this microglial response.

Several UREs (upstream regulatory elements) exert control over the Pu.1 gene locus. These UREs can be directly bound by PU.1, which functions as the auto-regulatory mechanism of this transcription factor (Li et al., 2001; Okuno et al., 2005). Indeed, three distinct PU.1 target sites were observed at $-14 \mathrm{~kb},-12 \mathrm{~kb}$ and $-10 \mathrm{~kb}$ of the Pu.1 locus, proving the quality of our ChIP-Seq analysis (Fig. 4D). Interestingly, we also noticed the presence of an IRF8 target site at $-10 \mathrm{~kb}$ of the Pu.1 locus, raising the possibility that IRF8 might feedback regulate PU.1 expression in microglial activation. We therefore bred $\mathrm{Cx} 3 \mathrm{cr} 1^{\text {CreERT2-EYFP/+}}$; Irf $8^{\mathrm{fl} / \mathrm{fl}}$ mice to achieve the microglia-specific deletion of IRF8 in adult mice, circumventing the developmental defect of microglia in Irf8 ${ }^{-/-}$mice (Kierdorf and Prinz, 2013; Kierdorf et al., 2013). As expected, while there was significant IRF8 up-regulation in the injured optic nerves of control $\mathrm{C} \times 3 \mathrm{Cr}^{+/+}$; Irf ${ }^{f l f l}$ mice, this IRF8 response was completely abolished in CX3cr1 ${ }^{\text {CreERT2-EYFP/+; Irf8 }}{ }^{\text {flffl }}$ mice, showing the efficient deletion of IRF8 expression (Fig. 4E and 4F). Moreover, the enhanced expression of PU.1 was largely mitigated in Cx3cr1 ${ }^{\text {CreERT2-EYFPl+} ; ~ I r f 8 ~}{ }^{f l / f l}$ mice (Fig. 4E and 4F). These results have together uncovered the cross-regulation of IRF8 


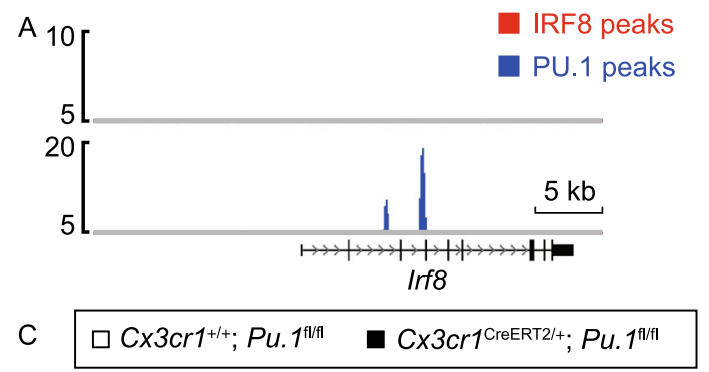

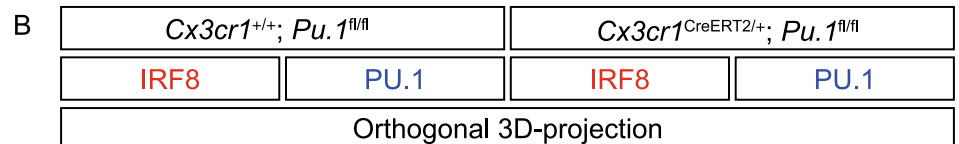
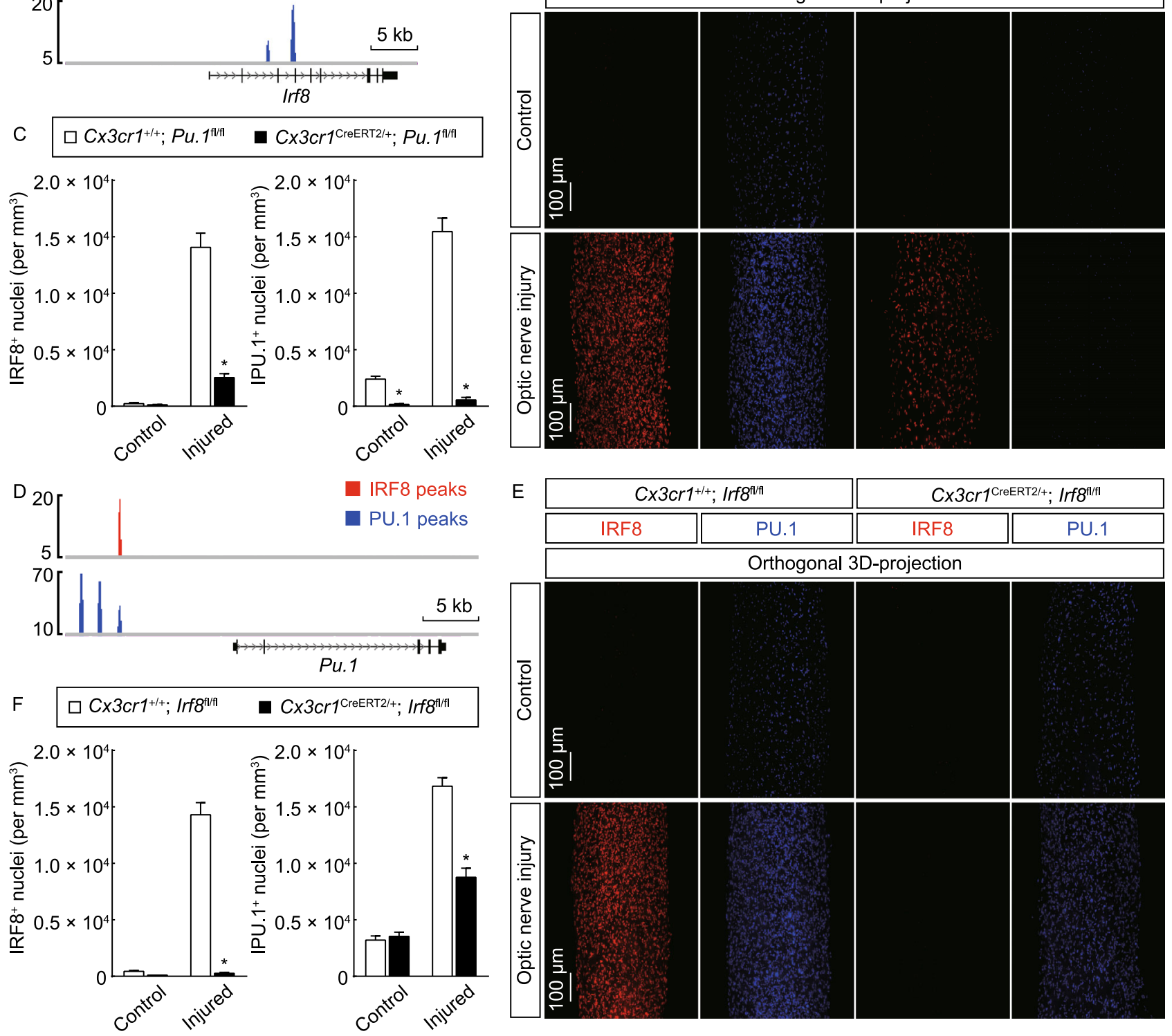

Figure 4. Cross-regulation of IRF8 and PU.1 in microglial activation. (A) PU.1 directly targets IRF8 expression in microglial activation. IRF8 and PU.1 target sites at the gene locus of Irf8 are shown. (B and C) PU.1 regulates IRF8 expression. Cx3cr1 ${ }^{+/+}$; $P u .1^{f l f f l}$ vs. $\mathrm{Cx} 3 \mathrm{cr}^{\mathrm{CreERT} 2 /+} ; \mathrm{Pu} .1^{\mathrm{flffl}}$ mice were treated with 4-hydroxytamoxifen to induce the Cre-recombinase activity and then subjected to optic nerve injury. The control and injured nerves were processed for the whole-tissue immunolabeling of IRF8 or PU.1 and imaged on the lightsheet microscope. (B) Representative orthogonal 3D-projection images of the optic nerves. (C) Density of IRF8 ${ }^{+}$or PU. $1^{+}$nuclei. $n=3$, mean \pm SEM, ${ }^{*} P<0.01$ (ANOVA test). (D) IRF8 directly targets PU. 1 expression in microglial activation. IRF8 and PU.1 target sites at the UREs of Pu.1 gene locus are shown. (E and F) IRF8 feedback regulates PU.1 expression. $\mathrm{C} \times 3 \mathrm{Cr}^{+/+}$; Irff ${ }^{f / f l}$ vs. $\mathrm{C} \times 3 \mathrm{cr} 1^{\mathrm{CreERT} 2 /+}$; Irff ${ }^{f / f l}$ mice were treated with 4-hydroxytamoxifen to induce the Cre-recombinase activity and then subjected to optic nerve injury. The control and injured nerves were processed for the whole-tissue immunolabeling of IRF8 or PU.1 and imaged on the lightsheet microscope. (E) Representative orthogonal 3D-projection images of the optic nerves. (F) Density of IRF8 ${ }^{+}$or PU. $1^{+}$nuclei. $n=3$, mean \pm SEM, ${ }^{*} P<0.01$ (ANOVA test). 
and PU.1 in microglial activation under the neurodegenerative condition.

\section{IRF8 and PU.1 cooperatively dictate microglial activation}

To further understand the transcriptional mechanism of microglial activation, IRF8 and PU.1 target sites in the genome were analyzed in detail. A total of 331 genes were identified to contain the IRF8-PU.1 co-target sites in the coding and/or regulatory regions (Table S1). The Gene Ontology (GO) enrichment analysis of these co-targeted genes revealed significant enrichment in several biological processes critical for microglial activation (Colonna and Butovsky, 2017; Li and Barres, 2018), e.g., cellular metabolism, cell differentiation, immune process, cell morphogenesis, chemotaxis and phagocytosis (Fig. 5A). In addition, the JNK and NF-KB signaling pathways, which are known for their essential roles in immune response (Vallabhapurapu and Karin, 2009; Arthur and Ley, 2013; Zhang et al., 2017), were also enriched (Fig. 5A).

To verify the cooperative transcriptional action of IRF8 and PU.1, we chose the NF-KB pathway as an example. The NF$\mathrm{KB}$ pathway was strongly activated in the microglial response to neurodegeneration, as assessed by the increased $\mathrm{p}-\mathrm{I} \mathrm{KB} \alpha$ immunostaining (Figs. 5B and S4C). Several key signaling components of the NF-KB pathway, i.e., Ripk2, Tak1, Ikbkb and Nfkb1, were identified to harbor the IRF8-PU.1 co-target sites containing the IRF-ETS composite motifs (Fig. 5C). We performed the electrophoretic mobility shift assay (EMSA) to biochemically examine the assembly of a ternary complex of recombinant IRF8 and PU.1 proteins with the composite-motif DNA. IRF8 or PU.1 alone exhibited weak or undetectable interaction with the DNA probes of Ripk2, Tak1, Ikbkb or Nfkb1 (Fig. 5D). Importantly, simultaneous inclusion of IRF8 and PU.1 proteins in the in vitro reactions led to the synergetic binding of IRF8 and PU.1 with each probe tested (Fig. 5D), showing the cooperative action of the two transcription factors in targeting the IRF-ETS composite motif. To further prove the specificity of this ternary-complex formation, we performed the supershift assay of the composite motif/IRF8/PU.1 complex with anti-IRF8 or anti-PU.1 antibody. Addition of the control IgG exhibited no effect on the ternary complex with Ripk2 or Tak1 probes (Fig. 5E). In contrast, addition of anti-IRF8 or antiPU.1 lgG both resulted in the supershift of the composite motif/ IRF8/PU.1 ternary complex to the higher molecular weight (Fig. 5E), confirming the presence of IRF8 and PU.1 proteins in the biochemically-assembled complex. These results have demonstrated the cooperative action of IRF8 and PU.1 in targeting the composite IRF-ETS motif in microglial activation.

To determine the central role of this transcriptional mechanism of IRF8 and PU.1 in microglial activation, a collection of microglial activation-related genes were chosen, e.g., the common marker for microglial activation (Iba1), immune process (Ly86, Mpeg1 and C1qb), cell morphogenesis (Lyn and Lpxn), phagocytosis (Ncf4, Ctsb and $\mathrm{Hexb}$ ) and cell differentiation (Oct2). We showed that all of these genes contained the IRF8-PU.1 co-target sites with the composite IRF-ETS motifs (Figs. 6A and S5A). Expression levels of the genes were significantly up-regulated in the injured optic nerves of $\mathrm{Cx} 3 \mathrm{Cr}^{+/+}$; Irf ${ }^{f l / f l}$ mice, but such

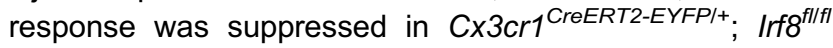
mice (Fig. 6B). In line with this suppression of microglial activation-related genes, the microglial proliferation was also inhibited in $C \times 3 \mathrm{Cr} 1^{\mathrm{CreERT2}-E Y F P /+}$; Irf8 $8^{\mathrm{fllfl}}$ mice compared to $\mathrm{C} \times 3 \mathrm{Cr}^{+/+} ;$Irf ${ }^{f l / f l}$ mice, as assessed by the 3D fluorescence imaging of $\mathrm{Ki}^{+} 7^{+}$nuclei (Fig. 6C and 6F). Accordingly, while the density of microglial population in the control optic nerves was indistinguishable between $\mathrm{C} \times 3 \mathrm{Cr}^{+/+} ; \quad \operatorname{Irf} 8^{\mathrm{fllfl}}$ vs.

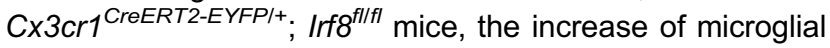
population was largely blunted in the injured optic nerves of CX3cr1 ${ }^{\text {CreERT2-EYFP/+ }}$; Irf8 ${ }^{\text {fl/fl }}$ mice (Fig. 6D and 6G). In addition, the IRF8-deficient microglia exhibited a stumpy morphology with fewer cellular processes in the injured optic nerves (Fig. 6E), likely reflecting the fact that IRF8 directly targets the pathway of cell morphogenesis as identified by the $\mathrm{GO}$ analysis (Fig. 5A).

Strikingly, microglial deletion of PU.1 in Cx3cr1 ${ }^{\text {CreERT2-EYFP/+; }}$ $P u .1^{f l f f l}$ resulted in the outcomes largely resembling those observed in $C \times 3 c r 1^{C r e E R T 2-E Y F P /+}$; Irf8 ${ }^{\text {fl/fl }}$ mice, i.e., the upregulation of microglial activation-related genes was abolished in the injured optic nerves of $C \times 3 c r 1^{\text {CreERT2-EYFP/+; }}$ $P u .1^{f l / f l}$ mice (Fig. 7A), supporting the cooperative action of IRF8 and PU.1 in this microglial response. Also, the microglial proliferation, as well as the increase of microglial population, in response to neurodegeneration was significantly inhibited in Cx3cr1 $1^{\text {CreERT2-EYFP/+} ; P u .1^{f l f l}}$ mice (Fig. 7B, 7C, 7E and 7F). Moreover, the PU.1-deficient microglia showed the stumpy cell morphology similar to that observed with the IRF8-deficient microglia (Fig. 7D and Movie S4). Taken together, these results have revealed the central transcriptional mechanism of microglial activation under neurodegenerative condition.

\section{DISCUSSION}

In this study, we have exploited the 3D fluorescence imaging to investigate the microglial response to neurodegeneration. Notably, this imaging technique is also applicable to assess other neuropathological processes, e.g., the degeneration of optic axons or the proliferation of oligodendrocytes. Moreover, the 3D fluorescence imaging could be readily extended to examine a variety of glial events in neural tissues beyond the optic nerves, e.g., brain and spinal cord, which would certainly assist in the comprehensive characterization of different CNS diseases.

Previous studies showed that PU.1 is required for the development of myeloid lineage cells, including microglia, in early embryonic stage (Scott et al., 1994; McKercher et al., 1996; Kierdorf et al., 2013). However, whether PU.1 might exert any function in microglial activation in the adulthood 


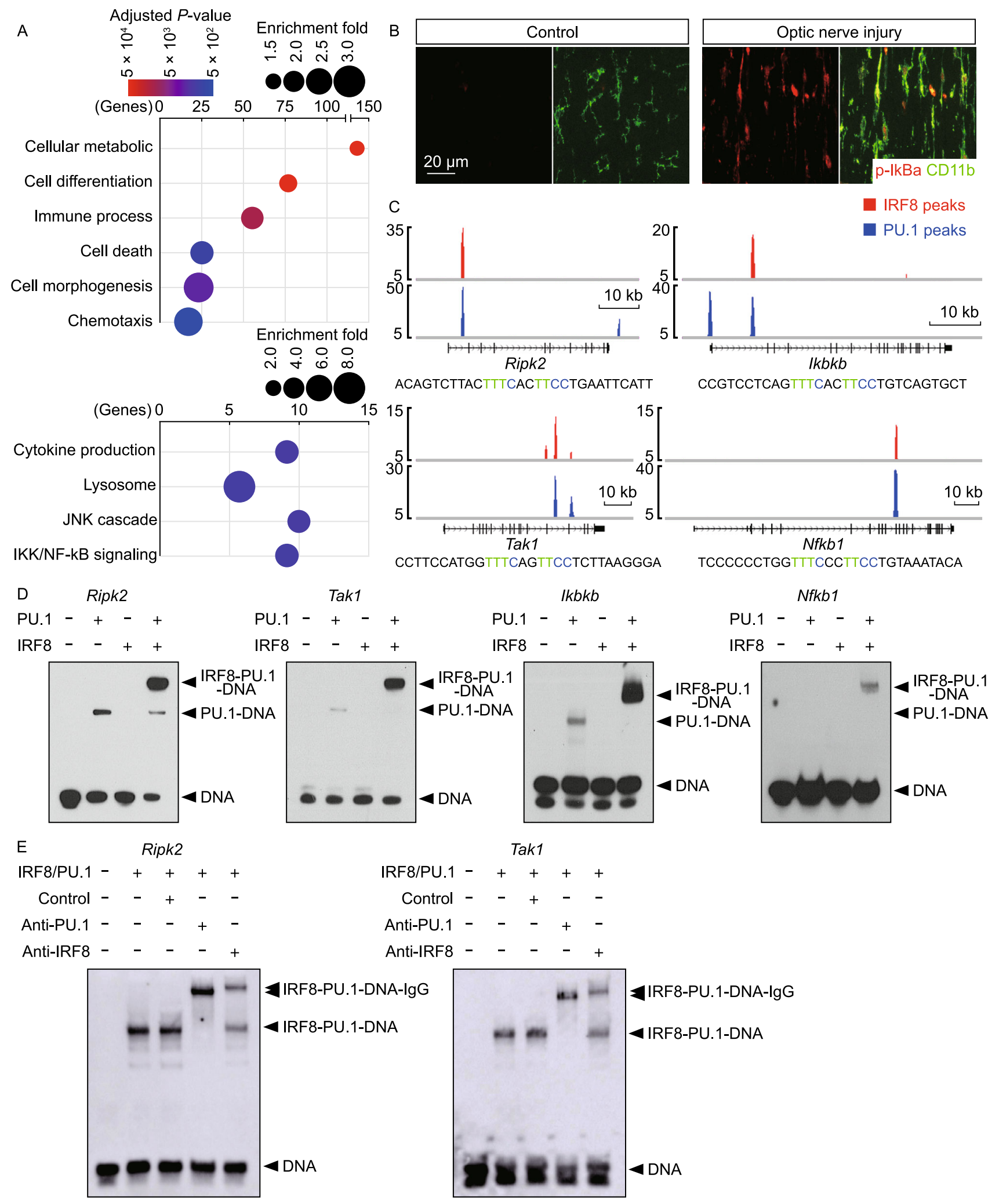


Figure 5. IRF8 and PU.1 cooperatively target microglial activation-related genes. (A) IRF8 and PU.1 cooperatively regulate microglial activation-related genes. The genes that contained the IRF8-PU.1 co-target sites were subjected to GO enrichment analysis. Enriched biological processes and signaling pathways are shown. (B) Activation of the NF-kB pathway in microglial activation. Wild-type mice were subjected to optic nerve injury. The control and injured nerves were examined by immunohistochemistry for $\mathrm{p}-\mathrm{IKB} \alpha$ and CD11b. (C) IRF8 and PU.1 directly target the key signaling components of the NF-kb pathway. IRF8 and PU.1 target sites at the indicated gene loci are shown. The sequence of the DNA probe derived from the IRF8-PU.1 co-target site at each locus is included, with the IRFETS composite motif highlighted. ( $D$ and $E$ ) Biochemical assembly of the ternary complex of the composite-motif DNA with IRF8 and PU.1 proteins. (D) Synergetic binding of IRF8 and PU.1 to the composite-motif DNA, derived from the gene loci of Ripk2, Tak1, Ikbkb or Nfkb1, was examined by EMSA. (E) Supershift of the composite-motif DNA/IRF8/PU.1 ternary complex with anti-IRF8 or anti-PU.1 antibody.

had been unexplored. We have found that PU.1 is highly upregulated in the microglial response to neurodegeneration. Through the microglia-specific deletion of PU.1 in adult mice, we have demonstrated its key post-developmental role in the transition of resting microglia to their activated state. In addition, PU. 1 does not act by itself in this process but rather cooperates with IRF8. Again, the genetic approach of microglia-specific deletion of IRF8 in adult mice has substantiated the critical post-developmental role of IRF8 in microglial activation, circumventing the reported developmental deficit of microglia in Irf8 $8^{-/-}$mice (Kierdorf and Prinz, 2013; Kierdorf et al., 2013).

Importantly, we have elucidated the transcriptional mechanism of IRF8 and PU.1 by profiling their genomic landscapes in activated primary microglia for the first time in the field. We have revealed that IRF8 and PU.1 cross-regulate each other in the process of microglial activation, i.e., PU.1 promotes IRF8 expression by directly targeting the gene locus of Irf8, and conversely, IRF8 regulates PU.1 expression through one of the UREs of the Pu. 1 locus. It appears plausible that this positive feedback between IRF8 and PU.1 would effectively sustain their highly enhanced expression, which is essential for the process of microglial activation. Moreover, we have identified that IRF8 and PU.1 dictates the microglial response by cooperatively targeting a large collection of microglial activation-related genes, e.g., the key signaling components of the NF-kB pathway, through the composite IRF-ETS motifs that are enriched at those gene loci. Our work has therefore uncovered the central role of the transcriptional module comprising IRF8, PU.1 and their composite motif in the microglial response to neurodegeneration (Fig. S6).
Given the broad involvement of microglial activation in the CNS diseases, it is a tempting possibility that the transcriptional mechanism we elucidated could also be in action under neuropathological conditions beyond the traumatic injury-induced neurodegeneration. For instance, IRF8 is up-regulated and contributes to the disease progression in the mouse model of experimental autoimmune encephalomyelitis (Yoshida et al., 2014). Also, IRF8 deficiency ameliorates the symptoms in the mouse model of neuropathic pain (Masuda et al., 2012; Inoue and Tsuda, 2018). In light of our current findings, whether PU.1 might be involved, and cooperate with IRF8, in such disease scenarios needs to be determined. Furthermore, future research is warranted to explore the upstream signaling pathway(s) that triggers the up-regulation of IRF8 and PU. 1 in response to different neuropathological cues.

In summary, our study has uncovered the central transcriptional mechanism that governs the microglial activation under neurodegenerative condition, which would advance our in-depth understanding of the beneficial and detrimental functions of microglia in the CNS diseases. Also, manipulation of this transcriptional mechanism of IRF8 and PU.1 might become a novel entry point for treatment of divergent neuropathological conditions.

\section{MATERIALS AND METHODS}

\section{Animal information}

All the experimental procedures in mice were performed in compliance with the protocols approved by the Institutional Animal Care and Use Committees (IACUC) of Peking University and Tsinghua University.

The mice utilized in the experiments were 2 to 4 months old. C57BL/6 wildtype mice were purchased from Charles River International. Sarm1 ${ }^{-1-}$ (JAX 018069, RRID:IMSR_JAX:018069), Aldh1/1GFP (MMRRC 011015-UCD, RRID:MMRRC_011015-UCD),

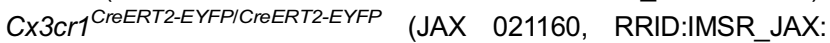
021160), Ccr2 ${ }^{-/-}$(JAX 017586, RRID:IMSR_JAX:017586), Rosa26LSL-tdTomato (JAX 007914, RRID:IMSR_JAX:007914), Irff ${ }^{f l f l}$ (JAX 014175, RRID:IMSR_JAX:014175) and Pu. $1^{\text {fllfl }}$ (JAX 006922, RRID: IMSR_JAX:006922) were purchased and bred in-house to generate the littermates for experiments.

Intravitreal injection of the tdTomato-expressing AAV2 was performed as previously described (Yang et al., 2015). The mice were anesthetized, and a customized 33-gauge, $30^{\circ}$-beveled needle attached to a Hamilton syringe was passed through the sclera into the vitreous. For the purpose of sparse labeling of RGCs, $1 \times 10^{3}$ to $5 \times 10^{3}$ transduction units of AAV2 were delivered, which transduced approximately 100 to 500 RGCs in each retina.

Traumatic injury of the optic nerves was performed as previously described (Yang et al., 2015). The mice of indicated conditions were anesthetized, and the topical antibiotic ointment was applied to the eyes. An incision was made on the superior conjunctiva of the left eye, and the optic nerve was exposed by a pair of blunt forceps. The crush injury was performed for $5 \mathrm{~s}$ using a pair of fine-tip forceps (Fine Science Tools) at approximately $1 \mathrm{~mm}$ distal from the eyeball. 
A
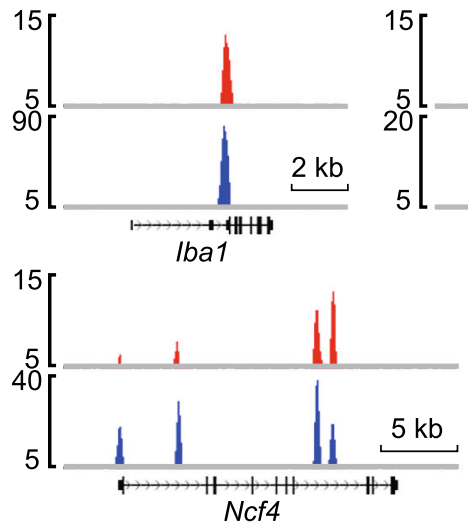
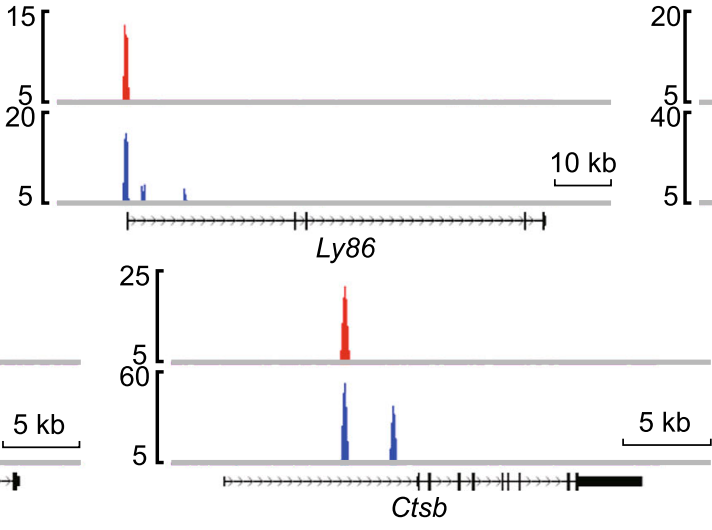

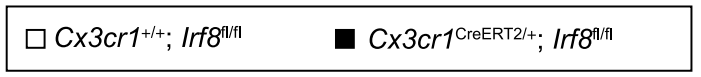

B
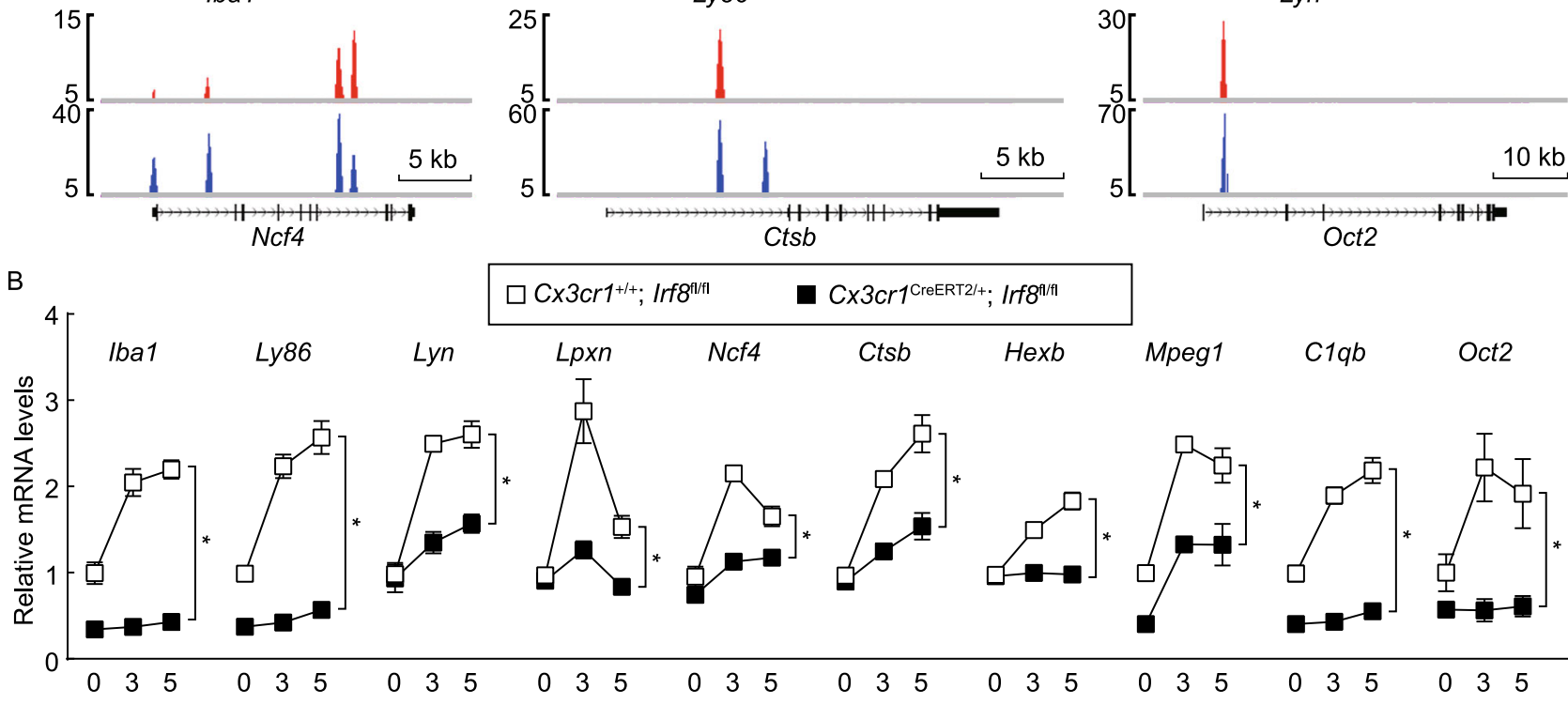

C

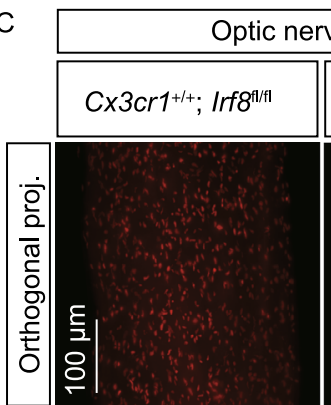

E

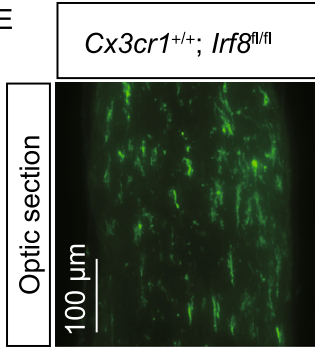

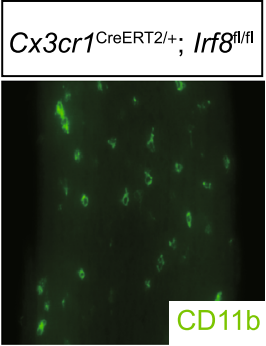
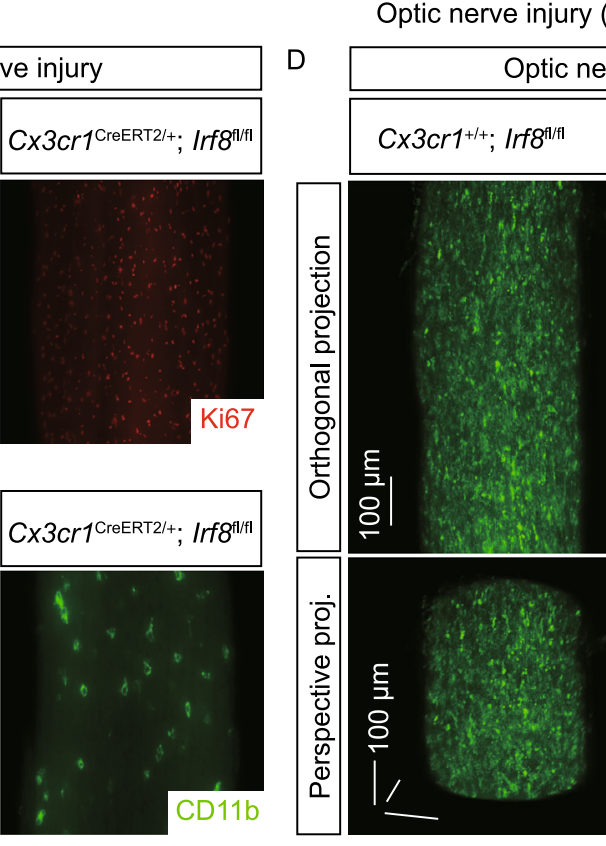

(Day)
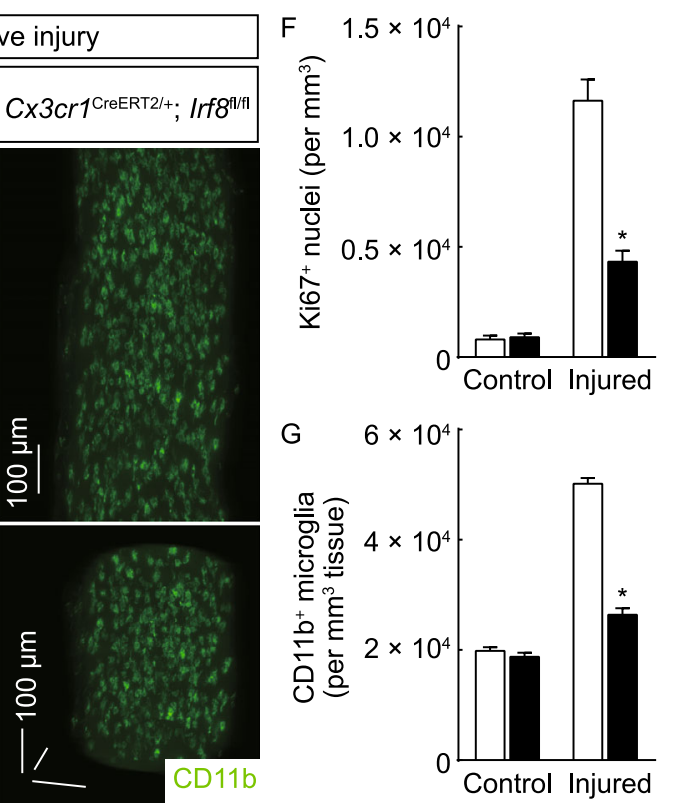

Figure 6. Post-developmental IRF8 is required for microglial activation. (A) IRF8 and PU.1 cooperatively target microglial activation-related genes. IRF8 and PU.1 target sites at the indicated gene loci are shown. (B-G) Post-developmental IRF8 is required

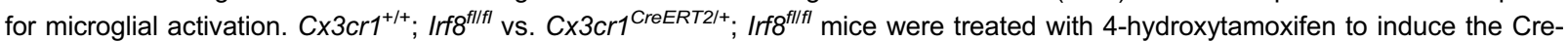
recombinase activity and then subjected to optic nerve injury. (B) Expression levels of the indicated genes in the optic nerves were examined by qPCR analysis. $n=4$, mean \pm SEM, ${ }^{*} P<0.01$ (ANOVA test). ( $C$ to $G$ ) The control and injured nerves were processed for the whole-tissue immunolabeling of Ki67 (C and F) or CD11b (D-G) and imaged on the lightsheet microscope. (C) Representative orthogonal 3D projections of the Ki67-immunolabeled injured nerves. (F) Density of Ki67 ${ }^{+}$nuclei. $n=3$, mean $\pm \mathrm{SEM}$, ${ }^{*} P<0.01$ (ANOVA test). (D) Representative orthogonal (upper panels) or perspective (lower panels) 3D projections of the CD11bimmunolabeled injured nerves. (E) Representative optical sections of the CD11b-immunolabeled injured nerves. (G) Density of CD $11 \mathrm{~b}^{+}$microglia. $n=4$, mean $\pm \mathrm{SEM},{ }^{*} P<0.01$ (ANOVA test). 

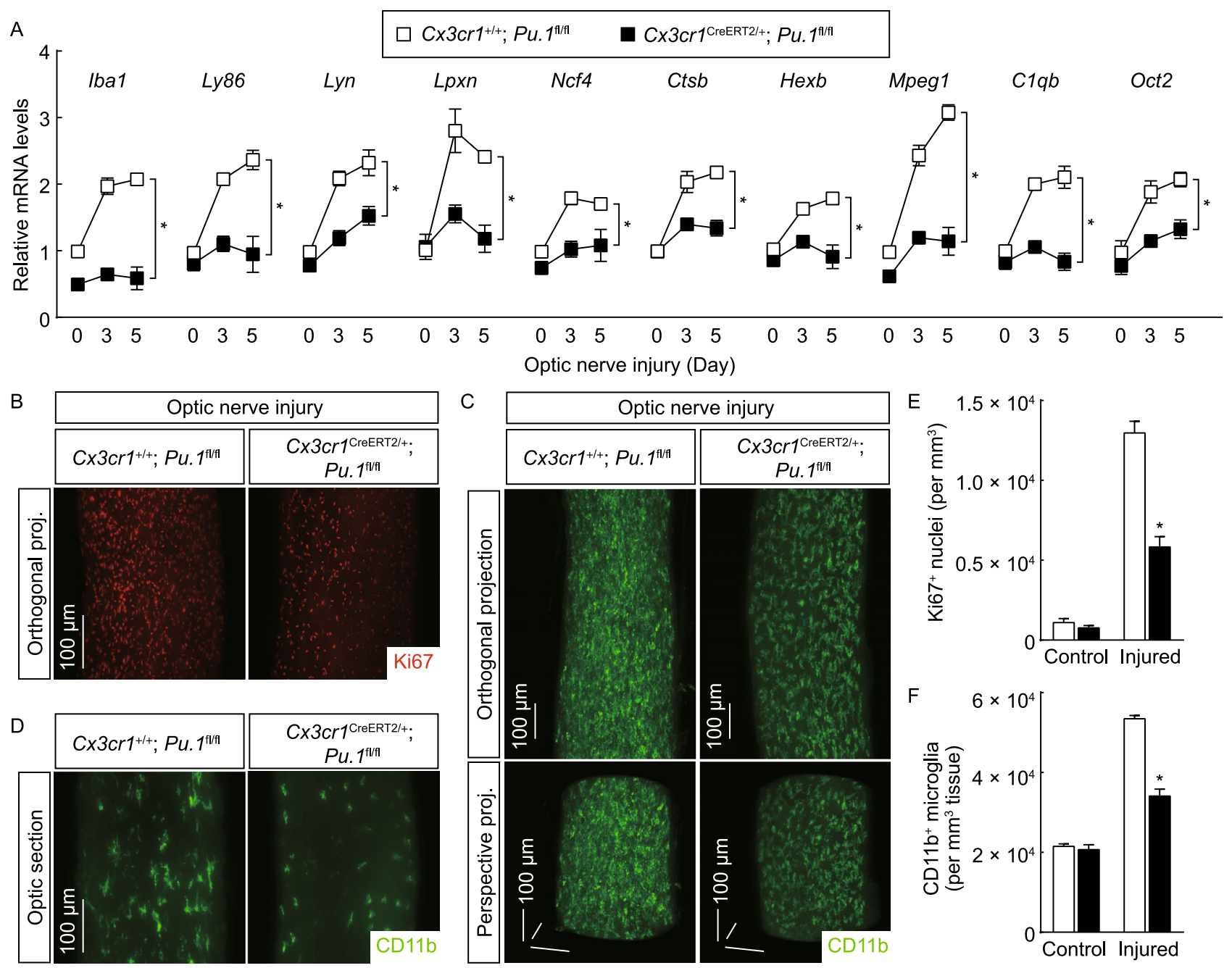

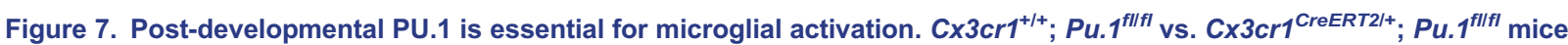
were treated with 4-hydroxytamoxifen to induce the Cre-recombinase activity and then subjected to optic nerve injury. (A) Expression levels of the indicated genes in the optic nerves were examined by qPCR analysis. $n=4$, mean \pm SEM, ${ }^{*} P<0.01$ (ANOVA test). (B-F) The control and injured nerves were processed for the whole-tissue immunolabeling of Ki67 (B and E) or CD11b (C, D and F) and imaged on the lightsheet microscope. (B) Representative orthogonal 3D projections of the Ki67-immunolabeled injured nerves. (E) Density of Ki67 ${ }^{+}$nuclei. $n=3$, mean \pm SEM, ${ }^{*} P<0.01$ (ANOVA test). (C) Representative orthogonal (upper panels) or perspective (lower panels) 3D projections of the CD11b-immunolabeled injured nerves. (D) Representative optical sections of the CD11b-immunolabeled injured nerves. (F) Density of CD11 ${ }^{+}$microglia. $n=4$, mean \pm SEM, ${ }^{*} P<0.01$ (ANOVA test).

For the parabiosis procedure, each pair of mice was housed together for 1 week before the surgery. The mice were anesthetized, and the skin on one side of each mouse was shaved and prepared with iodine and alcohol. A longitudinal incision was made along the side of each mouse, and the skin was carefully separated from the underlying connective tissues. A longitudinal incision of approximately $10 \mathrm{~mm}$ was then made on the exposed peritoneum of each mouse. The incision sites of the two mice were sutured together to establish the connection of vascular systems. In addition, the scapulae on the incision sides of the mice were sutured together to help hold the parabiotic pair. The skin incisions of the mice were closed together by surgical staples. The mice were utilized for experiments 4 weeks after the procedure.

To induce the Cre-recombinase activity in mice of the indicated genotypes, 4-hydroxytamoxifen (Sigma) was formulated in DMSO/ Kolliphor-EL/5\% sucrose (1:3:6) and administered daily to the mice at $20 \mathrm{mg} / \mathrm{kg}$ of body weight via oral gavage for 6 days. To circumvent the issue of microglial death observed around two weeks after the Cre-mediated genetic deletion of PU.1, Cx3cr1 ${ }^{\mathrm{CreERT2/+}} ; \mathrm{Pu} .1^{\mathrm{flffl}}$ mice were subjected to optic nerve injury immediately after 4-hydroxytamoxifen treatment. Otherwise, $\mathrm{Cx} 3 \mathrm{Cr} 1^{\mathrm{CreERT} 2 /+}$; Irff ${ }^{f / f l}$ mice were subjected to optic nerve injury at 4 weeks after 4-hydroxytamoxifen treatment. 


\section{Antibodies}

Primary antibodies used in the experiments were rat anti-PECAM1 (BD Biosciences \#553370, RRID:AB_394816), rat anti-LYVE1 (Thermo Fisher Scientific \#14-0443-82, RRID:AB_1633414), chicken anti-GFP (Aves Labs \#GFP-1010, RRID:AB_2307313), rat anti-CD11b (Biolegend \#101202, RRID:AB_312785), rabbit antiRFP (Rockland \#600-401-379, RRID:AB_2209751), mouse antiIba1 (Millipore \#MABN92, RRID:AB_10917271), rabbit anti-Olig2 (Millipore \#AB9610, RRID:AB_10141047), goat anti-IRF8 (Santa Cruz \#sc-6058, RRID:AB_649510), rabbit anti-PU.1 (Cell Signaling \#2258, RRID:AB_10693421), rabbit anti-Ki67 (Millipore \#AB9260, RRID:AB_2142366) and mouse anti-p-IkBa (Cell Signaling \#9246, RRID:AB_2267145). Alexa Fluor-conjugated secondary antibodies were from Thermo Fisher Scientific.

\section{D fluorescence imaging}

The 3D fluorescence imaging of optic nerves was optimized based on the reported iDISCO technique (Renier et al., 2014). The mice were perfused with $\mathrm{PBS} / 50 \mu \mathrm{g} / \mathrm{mL}$ heparin followed by $\mathrm{PBS} / 1 \%$ $\mathrm{PFA} / 10 \%$ sucrose $/ 50 \mu \mathrm{g} / \mathrm{mL}$ heparin. The optic nerves were dissected out and post-fixed in PBS/0.5\% PFA at room temperature for $2 \mathrm{~h}$. The tissues were washed with PBS at room temperature for 30 min and permeabilized with PBS $/ 0.1 \%$ TritonX-100/0.1\% deoxycholate/10\% DMSO/10 mmol/L EDTA ( $\mathrm{pH} \mathrm{8.0)}$ ) at room temperature for $6 \mathrm{~h}$. The tissues were blocked with PBS/0.2\% TritonX-100/10\% DMSO/10 mmol/L EDTA ( $\mathrm{pH} 8.0) / 5 \%$ normal donkey serum at room temperature overnight. The tissues were immunolabeled with the indicated primary antibodies diluted (1:500) in PBS/0.2\% Tween-20/ $10 \mu \mathrm{g} / \mathrm{mL}$ heparin $/ 5 \% \mathrm{DMSO} / 5 \%$ normal donkey serum at room temperature for $48 \mathrm{~h}$ and washed with PBS/0.2\% Tween-20/10 $\mu \mathrm{g} /$ $\mathrm{mL}$ heparin at room temperature for $2 \mathrm{~h}$ five times. The tissues were then immunolabeled with the corresponding Alexa Fluor-conjugated secondary antibodies diluted $(1: 1,000)$ in PBS/0.2\% Tween-20/10 $\mu \mathrm{g} / \mathrm{mL}$ heparin $/ 5 \% \mathrm{DMSO} / 5 \%$ normal donkey serum at room temperature for $24 \mathrm{~h}$ and washed with PBS/0.2\% Tween-20/10 $\mu \mathrm{g} / \mathrm{mL}$ heparin at room temperature for $2 \mathrm{~h}$ five times.

For the procedure of tissue clearing, the immunolabeled optic nerves were embedded in $0.8 \%$ agarose-blocks prepared in PBS. The tissue blocks were dehydrated at room temperature in $20 \%$ methanol (diluted in $\mathrm{ddH}_{2} \mathrm{O}$ ) for $2 \mathrm{~h}, 40 \%$ methanol for $2 \mathrm{~h}, 60 \%$ methanol for $2 \mathrm{~h}, 80 \%$ methanol for $2 \mathrm{~h}$, and $100 \%$ methanol for $1 \mathrm{~h}$ twice. The tissue blocks were incubated with the mixture of dichloromethane and methanol (2:1) for $2 \mathrm{~h}$ and then with $100 \%$ dichloromethane for $30 \mathrm{~min}$ twice. The tissue blocks were cleared with $100 \%$ dibenzyl-ether for $12 \mathrm{~h}$ twice to become ready for the lightsheet fluorescence imaging.

The tissue blocks were imaged on the LaVisionBiotec Ultramicroscope II equipped with the sCMOS camera (Andor $\mathrm{Neo}$ ) and the $2 \times /$ NA0.5 objective (MVPLAPO) covered with the 4-mm-workingdistance dipping-cap. Version 144 of the ImSpector Microscope Controller software was supported by LaVisionBiotec. The tissue blocks were immersed in the chamber filled with $100 \%$ dibenzylether. For the imaging at $12.6 \times$ effective magnification $(6.3 \times$ zoom), each tissue block was scanned by a single lightsheet (middle position) with a step size of $1 \mu \mathrm{m}$. The image stacks were acquired by the continuous lightsheet scanning method without the contrastblending algorithm.

Imaris (http://www.bitplane.com/imaris/imaris) was used to reconstruct the image stacks obtained from the lightsheet imaging. For display purposes in the figures and movies, a gamma correction of 1.2-1.6 was applied to the raw data (Renier et al., 2014). Movies of the image stacks were generated with the frame rate of $30 \mathrm{fps}$. Perspective or orthogonal 3D projections of the image stacks were generated as indicated for the representative images shown in figures.

\section{ChIP-Seq analysis}

To determine the transcriptional landscape of IRF8 and PU.1, the procedure of chromatin immunoprecipitation (ChIP) was optimized for the optic nerve tissues. The wildtype mice were subjected to optic nerve injury, and 70 injured optic nerves were acutely harvested. The nerve tissues were immediately washed once in ice-cold PBS and homogenized in ice-cold PBS containing protease inhibitor cocktail (Roche) with a Dounce homogenizer. A final concentration of $1 \%$ PFA was added, and the tissue lysates were cross-linked at room temperature for $10 \mathrm{~min}$. The cross-linking reaction was stopped by a final concentration of $125 \mathrm{mmol} / \mathrm{L}$ glycine at room temperature for $5 \mathrm{~min}$, followed by centrifugation at $1,000 \times g$ at $4{ }^{\circ} \mathrm{C}$ for $5 \mathrm{~min}$ to collect the nuclei. The pellet of nuclei was washed with $5 \mathrm{mmol} / \mathrm{L}$ PIPES (pH 8.0)/85 mmol/L KCl/0.5\% NP-40/protease inhibitor cocktail at $4{ }^{\circ} \mathrm{C}$ for $10 \mathrm{~min}$, followed by centrifugation at $2,500 \times g$ at $4{ }^{\circ} \mathrm{C}$ for $5 \mathrm{~min}$. The pellet of nuclei was lysed with 50 $\mathrm{mmol} / \mathrm{L}$ Tris- $\mathrm{HCl}(\mathrm{pH} 8.1) / 10 \mathrm{mmol} / \mathrm{L}$ EDTA $(\mathrm{pH} 8.0) / 1 \%$ SDS/protease inhibitor cocktail at $4{ }^{\circ} \mathrm{C}$ for $10 \mathrm{~min}$. The chromatin was then sonicated on the Bioruptor Pico (30 s $+30 \mathrm{~s}$ cooling down, 20 cycles) to obtain the genomic DNA fragments ranging from $100 \mathrm{bp}$ to $500 \mathrm{bp}$, followed by centrifugation at $20,000 \times g$ at $10^{\circ} \mathrm{C}$ for $10 \mathrm{~min}$ to clear the supernatant.

The sheared chromatin was diluted (1:10) into $16.7 \mathrm{mmol} / \mathrm{L}$ Tris$\mathrm{HCl}(\mathrm{pH} 8.1) / 16.7 \mathrm{mmol} / \mathrm{L} \mathrm{NaCl} / 1.2 \mathrm{mmol} / \mathrm{L}$ EDTA $(\mathrm{pH} 8.0) / 1.1 \%$ Triton $\mathrm{X}-100 / 0.01 \%$ SDS. Then, $10 \mu \mathrm{L}$ protein $\mathrm{A} / \mathrm{G}$ magnetic beads (Pierce) equilibrated with $10 \mathrm{mmol} / \mathrm{L}$ Tris- $\mathrm{HCl}(\mathrm{pH} 8.0) / 1 \mathrm{mmol} / \mathrm{L}$ EDTA $(\mathrm{pH} 8.0) / 1 \mathrm{mg} / \mathrm{mL}$ BSA was added per $1 \mathrm{~mL}$ of the chromatin sample to pre-clear at $4{ }^{\circ} \mathrm{C}$ for $1 \mathrm{~h}$. Following the magnetic removal of protein A/G beads, $2 \mu \mathrm{g}$ of anti-IRF8 or anti-PU. 1 antibody was added per $1 \mathrm{~mL}$ of the pre-cleared chromatin sample and incubated at $4{ }^{\circ} \mathrm{C}$ overnight. Next, $10 \mu \mathrm{L}$ protein $\mathrm{A} / \mathrm{G}$ magnetic beads equilibrated with $10 \mathrm{mmol} / \mathrm{L}$ Tris-HCl $(\mathrm{pH} 8.0) / 1 \mathrm{mmol} / \mathrm{L}$ EDTA $(\mathrm{pH} 8.0) / 1$ $\mathrm{mg} / \mathrm{mL}$ BSA was added per $1 \mathrm{~mL}$ of the chromatin sample and incubated at $4{ }^{\circ} \mathrm{C}$ for $3 \mathrm{~h}$. The beads were washed at room temperature with $20 \mathrm{mmol} / \mathrm{L} \mathrm{Tris}-\mathrm{HCl}(\mathrm{pH} 8.1) / 150 \mathrm{mmol} / \mathrm{L} \mathrm{NaCl} / 2 \mathrm{mmol} /$ L EDTA (pH 8.0)/1\% Triton X-100/0.1\% SDS for $5 \mathrm{~min}, 20 \mathrm{mmol} / \mathrm{L}$ Tris-HCl $(\mathrm{pH} 8.1) / 500 \mathrm{mmol} / \mathrm{L} \mathrm{NaCl} / 2 \mathrm{mmol} / \mathrm{L}$ EDTA $(\mathrm{pH} 8.0) / 1 \%$ Triton X-100/0.1\% SDS for $5 \mathrm{~min}, 10 \mathrm{mmol} / \mathrm{L}$ Tris-HCl $(\mathrm{pH} 8.1) / 250$ $\mathrm{mmol} / \mathrm{L} \mathrm{LiCl} / 1 \mathrm{mmol} / \mathrm{L}$ EDTA $(\mathrm{pH} 8.0) / 1 \% \mathrm{NP}-40$ for $5 \mathrm{~min}$ and 10 $\mathrm{mmol} / \mathrm{L}$ Tris- $\mathrm{HCl}(\mathrm{pH} 8.0) / 1 \mathrm{mmol} / \mathrm{L}$ EDTA $(\mathrm{pH} 8.0)$ for $5 \mathrm{~min}$ twice. The immunoprecipitated chromatin was eluted with $100 \mathrm{mmol} / \mathrm{L}$ $\mathrm{NaHCO}_{3} / 1 \%$ SDS at room temperature for $15 \mathrm{~min}$ twice. A final concentration of $200 \mathrm{mmol} / \mathrm{L} \mathrm{NaCl}$ was added to the pooled eluate, and the cross-linked chromatin was reversed at $65^{\circ} \mathrm{C}$ overnight. $\mathrm{A}$ final concentration of $50 \mathrm{mmol} / \mathrm{L}$ Tris- $\mathrm{HCl}(\mathrm{pH} 6.5) / 10 \mathrm{mmol} / \mathrm{L}$ EDTA 
( $\mathrm{pH} 8.0) / 25 \mu \mathrm{g} / \mathrm{mL}$ proteinase $\mathrm{K}$ (New England Biolabs) was added to digest the proteins at $37{ }^{\circ} \mathrm{C}$ for $1 \mathrm{~h}$. The genomic DNAs were recovered by the QIAquick Purification Kit (Qiagen).

The immunoprecipitated genomic DNAs were sequenced on the Illumina Genome Analyzer II. The accession numbers for the ChIPSeq data are the Sequence Read Archive (https://www.ncbi.nIm. nih.gov/sra/docs/): SRR6963589 and SRR6963590. The sequencing data were mapped to the mouse genome $(\mathrm{mm} 10)$ with Bowtie2 (http://bowtie-bio.sourceforge.net/bowtie2/index.shtml/), and the fragments of mapping quality greater than 15 were included for the downstream analysis. IRF8 or PU.1 target sites were called by MACS2 (https://github.com/taoliu/MACS/downloads/). Analysis of the composite motif of IRF8 and PU.1 target sites was performed with MEME (http://meme-suite.org/). Gene Ontology (GO) enrichment analysis of the genes that contained the IRF8-PU.1 co-target sites was performed with PANTHER (http://pantherdb.org/).

Tissue processing and analyses

For the conventional immunohistochemistry, the mice of indicated conditions were perfused with PBS $/ 50 \mu \mathrm{g} / \mathrm{mL}$ heparin followed by PBS $/ 1 \%$ PFA $10 \%$ sucrose $/ 50 \mu \mathrm{g} / \mathrm{mL}$ heparin. The optic nerves were dissected out and post-fixed in PBS/0.5\% PFA at room temperature for $2 \mathrm{~h}$. The tissues were cryopreserved in $\mathrm{PBS} / 30 \%$ sucrose at $4{ }^{\circ} \mathrm{C}$ overnight, then processed for $5-\mu \mathrm{m}$ cryosectioning. The sections were immunostained with the indicated primary antibodies and corresponding Alexa Fluor-conjugated secondary antibodies, and then imaged by the fluorescence microscopy.

For the qPCR analysis of gene expression, the optic nerves were acutely dissected from the mice of indicated conditions. Total RNAs of the nerve tissues were extracted by the RNeasy Mini Kit (Qiagen), and then reverse-transcribed and analyzed by the SYBR Green Real-Time PCR Kit (Thermo Fisher Scientific).

Electrophoretic mobility shift assay

Mouse IRF8 and PU.1 cDNAs were cloned into the pcDNA3 vector with the Flag-tag. The plasmids were transfected into HEK293T cells, and recombinant IRF8 and PU.1 proteins were purified from the nuclear extracts of transfected cells with anti-Flag M2 Affinity Gel (Sigma). For each DNA probe, the sequence was derived from the IRF8-PU.1 co-target site at the indicated gene locus. The complementary pair of $5^{\prime}$-biotin-labeled primers were synthesized and annealed for each probe. The electrophoretic mobility shift assay (EMSA) was performed with the LightShift Chemiluminescent EMSA Kit (Thermo Fisher Scientific). For the supershift assay, anti-PU.1, anti-IRF8 or control IgG was added after the formation of the composite-motif DNA/IRF8/PU. 1 complex, and the biochemical reactions were incubated at $4{ }^{\circ} \mathrm{C}$ for $20 \mathrm{~min}$.

\section{Statistical methods}

To quantify the traumatic injury-induced neurodegeneration, the tdTomato-labeled axons were manually traced in the 3D-reconstructed image of each nerve tissue, with any sign of axonal fragmentation scored as degeneration. To quantify the density of Olig2 ${ }^{+}$ oligodendrocyte nuclei, three $100 \mu \mathrm{m} \times 100 \mu \mathrm{m} \times 100 \mu \mathrm{m}$ volumes were randomly selected along the 3D-reconstructed image of each nerve tissue, and the immunolabeled nuclei in each cubic volume were manually counted. To quantify the density of $\mathrm{Ki} 67^{+}, \mathrm{IRF}^{+}$or PU. $1^{+}$nuclei, four $100 \mu \mathrm{m} \times 100 \mu \mathrm{m} \times 100 \mu \mathrm{m}$ volumes were randomly selected along the 3D-reconstructed image of each nerve tissue, and the immunolabeled nuclei in each cubic volume were counted. To quantify the density of $\mathrm{Iba}^{+}$or $\mathrm{CD} 11 \mathrm{~b}^{+}$microglia, four $100 \mu \mathrm{m} \times 100 \mu \mathrm{m} \times 100 \mu \mathrm{m}$ volumes were randomly selected along the 3D-reconstructed image of each nerve tissue, and the immunolabeled microglia in each cubic volume were counted.

Student's $t$-test or ANOVA test was performed using GraphPad Prism (http://www.graphpad.com/scientific-software/prism). Statistical details of the experiments can be found in the figure legends.

\section{ACKNOWLEDGMENTS}

This study has been supported by State Key Laboratory of Membrane Biology, IDG/McGovern Institute for Brain Research, Center for Life Sciences and School of Life Sciences at Peking University, and National Natural Science Foundation of China to J. Yang (Grant Nos. 31522024 and 31771111).

\section{ABBREVIATIONS}

CNS, central nervous system; ChIP, chromatin immunoprecipitations; EMSA, electrophoretic mobility shift assay; ETS, E26 transformation-specific; GO, gene ontology; iDISCO, immunolabelingenabled three-dimensional imaging of solvent-cleared organs; IRF, interferon regulatory factor; URE, upstream regulatory element

\section{AUTHOR CONTRIBUTIONS}

J. Yang conceived and designed the project as the senior and corresponding author. N. Zhou, K. Liu, Y. Sun and Y. Cao performed the experiments and analyzed the results under the supervision of $\mathrm{J}$. Yang, N. Zhou, K. Liu, Y. Sun and Y. Cao contributed equally to this work.

\section{COMPLIANCE WITH ETHICS GUIDELINES}

Nan Zhou, Kaili Liu, Yue Sun, Ying Cao, and Jing Yang declare that they have no conflict of interest. All institutional and national guidelines for the care and use of laboratory animals were followed.

\section{OPEN ACCESS}

This article is distributed under the terms of the Creative Commons Attribution 4.0 International License (http://creativecommons.org/ licenses/by/4.0/), which permits unrestricted use, distribution, and reproduction in any medium, provided you give appropriate credit to the original author(s) and the source, provide a link to the Creative Commons license, and indicate if changes were made.

\section{REFERENCES}

Aguzzi A, Barres BA, Bennett ML (2013) Microglia: scapegoat, saboteur, or something else? Science 339:156-161 
Arthur JS, Ley SC (2013) Mitogen-activated protein kinases in innate immunity. Nat Rev Immunol 13:679-692

Biber K, Moller T, Boddeke E, Prinz M (2016) Central nervous system myeloid cells as drug targets: current status and translational challenges. Nat Rev Drug Discov 15:110-124

Colonna M, Butovsky O (2017) Microglia function in the central nervous system during health and neurodegeneration. Ann Rev Immunol 35:441-468

Colonna M, Wang Y (2016) TREM2 variants: new keys to decipher Alzheimer disease pathogenesis. Nat Rev Neurosci 17:201-207

Finsen B, Owens T (2011) Innate immune responses in central nervous system inflammation. FEBS Lett 585:3806-3812

Fu R, Shen Q, Xu P, Luo JJ, Tang Y (2014) Phagocytosis of microglia in the central nervous system diseases. Mol Neurobiol 49:1422-1434

Ginhoux F, Prinz M (2015) Origin of microglia: current concepts and past controversies. Cold Spring Harbor Perspect Biol 7:a020537

Ginhoux F, Lim S, Hoeffel G, Low D, Huber T (2013) Origin and differentiation of microglia. Front Cell Neurosci 7:45

Goldmann T, Wieghofer P, Muller PF, Wolf Y, Varol D, Yona S, Brendecke SM, Kierdorf K, Staszewski O, Datta M et al (2013) A new type of microglia gene targeting shows TAK1 to be pivotal in CNS autoimmune inflammation. Nat Neurosci 16:1618-1626

Heppner FL, Ransohoff RM, Becher B (2015) Immune attack: the role of inflammation in Alzheimer disease. Nat Rev Neurosci 16:358-372

Herz J, Filiano AJ, Smith A, Yogev N, Kipnis J (2017) Myeloid cells in the central nervous system. Immunity 46:943-956

Hollenhorst PC, Mclntosh LP, Graves BJ (2011) Genomic and biochemical insights into the specificity of ETS transcription factors. Ann Rev Biochem 80:437-471

Hong S, Dissing-Olesen L, Stevens B (2016) New insights on the role of microglia in synaptic pruning in health and disease. Curr Opin Neurobiol 36:128-134

Horiuchi M, Wakayama K, Itoh A, Kawai K, Pleasure D, Ozato K, Itoh $T$ (2012) Interferon regulatory factor 8/interferon consensus sequence binding protein is a critical transcription factor for the physiological phenotype of microglia. J Neuroinflamm 9:227

Inoue K, Tsuda M (2018) Microglia in neuropathic pain: cellular and molecular mechanisms and therapeutic potential. Nat Rev Neurosci 19:138-152

Kalin S, Heppner FL, Bechmann I, Prinz M, Tschop MH, Yi CX (2015) Hypothalamic innate immune reaction in obesity. Nat Rev Endocrinol 11:339-351

Kettenmann H, Hanisch UK, Noda M, Verkhratsky A (2011) Physiology of microglia. Physiol Rev 91:461-553

Kettenmann H, Kirchhoff F, Verkhratsky A (2013) Microglia: new roles for the synaptic stripper. Neuron 77:10-18

Kierdorf K, Prinz M (2013) Factors regulating microglia activation. Front Cell Neurosci 7:44

Kierdorf K, Erny D, Goldmann T, Sander V, Schulz C, Perdiguero EG, Wieghofer P, Heinrich A, Riemke P, Holscher C et al (2013) Microglia emerge from erythromyeloid precursors via Pu.1- and Irf8-dependent pathways. Nat Neurosci 16:273-280

Lall D, Baloh RH (2017) Microglia and C9orf72 in neuroinflammation and ALS and frontotemporal dementia. J Clin Invest 127:3250-3258
Li Q, Barres BA (2018) Microglia and macrophages in brain homeostasis and disease. Nat Rev Immunol 18:225-242

Li Y, Okuno Y, Zhang P, Radomska HS, Chen H, Iwasaki H, Akashi K, Klemsz MJ, McKercher SR, Maki RA et al (2001) Regulation of the PU.1 gene by distal elements. Blood 98:2958-2965

Lum FM, Low DK, Fan Y, Tan JJ, Lee B, Chan JK, Renia L, Ginhoux F, Ng LF (2017) Zika virus infects human fetal brain microglia and induces inflammation. Clin Infect Dis 64:914-920

Masuda T, Tsuda M, Yoshinaga R, Tozaki-Saitoh H, Ozato K, Tamura T, Inoue K (2012) IRF8 is a critical transcription factor for transforming microglia into a reactive phenotype. Cell Rep 1:334340

McKercher SR, Torbett BE, Anderson KL, Henkel GW, Vestal DJ, Baribault H, Klemsz M, Feeney AJ, Wu GE, Paige CJ et al (1996) Targeted disruption of the PU.1 gene results in multiple hematopoietic abnormalities. EMBO J 15:5647-5658

Meertens L, Labeau A, Dejarnac O, Cipriani S, Sinigaglia L, BonnetMadin L, Le Charpentier T, Hafirassou ML, Zamborlini A, CaoLormeau VM et al (2017) AxI mediates ZIKA virus entry in human glial cells and modulates innate immune responses. Cell Rep 18:324-333

Meyer-Luehmann M, Prinz M (2015) Myeloid cells in Alzheimer's disease: culprits, victims or innocent bystanders? Trends Neurosci 38:659-668

Michell-Robinson MA, Touil H, Healy LM, Owen DR, Durafourt BA, Bar-Or A, Antel JP, Moore CS (2015) Roles of microglia in brain development, tissue maintenance and repair. Brain 138:11381159

Minten C, Terry R, Deffrasnes C, King NJ, Campbell IL (2012) IFN regulatory factor 8 is a key constitutive determinant of the morphological and molecular properties of microglia in the CNS. PLoS ONE 7:e49851

Nayak D, Roth TL, McGavern DB (2014) Microglia development and function. Ann Rev Immunol 32:367-402

Neumann H, Kotter MR, Franklin RJ (2009) Debris clearance by microglia: an essential link between degeneration and regeneration. Brain 132:288-295

Okuno Y, Huang G, Rosenbauer F, Evans EK, Radomska HS, Iwasaki H, Akashi K, Moreau-Gachelin F, Li Y, Zhang P et al (2005) Potential autoregulation of transcription factor PU.1 by an upstream regulatory element. Mol Cell Biol 25:2832-2845

Osterloh JM, Yang J, Rooney TM, Fox AN, Adalbert R, Powell EH, Sheehan AE, Avery MA, Hackett R, Logan MA et al (2012) dSarm/Sarm1 is required for activation of an injury-induced axon death pathway. Science 337:481-484

Parkhurst CN, Yang G, Ninan I, Savas JN, Yates JR III, Lafaille JJ, Hempstead BL, Littman DR, Gan WB (2013) Microglia promote learning-dependent synapse formation through brain-derived neurotrophic factor. Cell 155:1596-1609

Perry VH, Holmes C (2014) Microglial priming in neurodegenerative disease. Nat Rev Neurol 10:217-224

Perry VH, Nicoll JA, Holmes C (2010) Microglia in neurodegenerative disease. Nat Rev Neurol 6:193-201

Prinz M, Priller J (2014) Microglia and brain macrophages in the molecular age: from origin to neuropsychiatric disease. Nat Rev Neurol 15:300-312 
Prinz M, Erny D, Hagemeyer N (2017) Ontogeny and homeostasis of CNS myeloid cells. Nat Immunol 18:385-392

Ransohoff RM (2016) How neuroinflammation contributes to neurodegeneration. Science 353:777-783

Ransohoff RM, Brown MA (2012) Innate immunity in the central nervous system. J Clin Invest 122:1164-1171

Ransohoff RM, Cardona AE (2010) The myeloid cells of the central nervous system parenchyma. Nature 468:253-262

Renier N, Wu Z, Simon DJ, Yang J, Ariel P, Tessier-Lavigne M (2014) iDISCO: a simple, rapid method to immunolabel large tissue samples for volume imaging. Cell 159:896-910

Rivest S (2009) Regulation of innate immune responses in the brain. Nat Reviews Immunol 9:429-439

Schafer DP, Stevens B (2015) Microglia function in central nervous system development and plasticity. Cold Spring Harbor Perspect Biol 7:a020545

Scott EW, Simon MC, Anastasi J, Singh H (1994) Requirement of transcription factor PU.1 in the development of multiple hematopoietic lineages. Science 265:1573-1577

Sharrocks AD (2001) The ETS-domain transcription factor family. Nat Rev Mol Cell Biol 2:827-837

Soto I, Howell GR (2014) The complex role of neuroinflammation in glaucoma. Cold Spring Harbor Perspect Med. https://doi.org/10. 1101/cshperspect.a017269

Stephan AH, Barres BA, Stevens B (2012) The complement system: an unexpected role in synaptic pruning during development and disease. Ann Rev Neurosci 35:369-389

Tay TL, Hagemeyer N, Prinz M (2016) The force awakens: insights into the origin and formation of microglia. Curr Opin Neurobiol 39:30-37

Tay TL, Savage JC, Hui CW, Bisht K, Tremblay ME (2017) Microglia across the lifespan: from origin to function in brain development, plasticity and cognition. j Physiol 595:1929-1945
Terry RL, Getts DR, Deffrasnes C, van Vreden C, Campbell IL, King NJ (2012) Inflammatory monocytes and the pathogenesis of viral encephalitis. J Neuroinflamm 9:270

Thaler JP, Choi SJ, Schwartz MW, Wisse BE (2010) Hypothalamic inflammation and energy homeostasis: resolving the paradox. Front Neuroendocrinol 31:79-84

Town T, Jeng D, Alexopoulou L, Tan J, Flavell RA (2006) Microglia recognize double-stranded RNA via TLR3. J immunol 176:38043812

Ulrich JD, Ulland TK, Colonna M, Holtzman DM (2017) Elucidating the role of TREM2 in Alzheimer's disease. Neuron 94:237-248

Valdearcos M, Xu AW, Koliwad SK (2015) Hypothalamic inflammation in the control of metabolic function. Ann Rev Physiol 77:131-160

Vallabhapurapu S, Karin M (2009) Regulation and function of NFkappaB transcription factors in the immune system. Ann Rev Immunol 27:693-733

Wolf SA, Boddeke HW, Kettenmann H (2017) Microglia in physiology and disease. Ann Rev Physiol 79:619-643

Yang J, Wu Z, Renier N, Simon DJ, Uryu K, Park DS, Greer PA, Tournier C, Davis RJ, Tessier-Lavigne M (2015) Pathological axonal death through a MAPK cascade that triggers a local energy deficit. Cell 160:161-176

Yoshida Y, Yoshimi R, Yoshii H, Kim D, Dey A, Xiong H, Munasinghe J, Yazawa I, O'Donovan MJ, Maximova OA et al (2014) The transcription factor IRF8 activates integrin-mediated TGF-beta signaling and promotes neuroinflammation. Immunity 40:187198

Zhang Q, Lenardo MJ, Baltimore D (2017) 30 years of NF-kappaB: a blossoming of relevance to human pathobiology. Cell 168:37-57

Zuchero JB, Barres BA (2015) Glia in mammalian development and disease. Development 142:3805-3809 